IASSNS-HEP-91/64

CALT-68-1764

HUTP-92/A003

December 1991

\title{
Quantum Hair on Black Holes
}

\author{
SIDNEY COLEMAN ${ }^{\star}$ \\ Lyman Laboratory of Physics \\ Harvard University \\ Cambridge, MA. 02138 \\ JOHN PRESKILL $^{\dagger}$ \\ Lauritsen Laboratory of High Energy Physics \\ California Institute of Technology \\ Pasadena, CA. 91125 \\ FRANK WILCZEK ${ }^{\ddagger}$ \\ School of Natural Sciences \\ Institute for Advanced Study \\ Olden Lane \\ Princeton, N.J. 08540
}

\footnotetext{
* Research supported in part by NSF grant PHY-87-14654

$\dagger$ Research supported in part by DOE grant DE-AC03-81-ER40050

$\ddagger$ Research supported in part by DOE grant DE-FG02-90ER40542
} 


\begin{abstract}
A black hole may carry quantum numbers that are not associated with massless gauge fields, contrary to the spirit of the "no-hair" theorems. We describe in detail two different types of black hole hair that decay exponentially at long range. The first type is associated with discrete gauge charge and the screening is due to the Higgs mechanism. The second type is associated with color magnetic charge, and the screening is due to color confinement. In both cases, we perform semi-classical calculations of the effect of the hair on local observables outside the horizon, and on black hole thermodynamics. These effects are generated by virtual cosmic strings, or virtual electric flux tubes, that sweep around the event horizon. The effects of discrete gauge charge are non-perturbative in $\hbar$, but the effects of color magnetic charge become $\hbar$-independent in a suitable limit. We present an alternative treatment of discrete gauge charge using dual variables, and examine the possibility of black hole hair associated with discrete global symmetry. We draw the distinction between primary hair, which endows a black hole with new quantum numbers, and secondary hair, which does not, and we point out some varieties of secondary hair that occur in the standard model of particle physics.
\end{abstract}




\section{Introduction}

\subsection{Challenges for the quantum theory of Black holes}

It has been claimed that existing results on the quantum mechanics of black holes require a modification of the fundamental laws of quantum mechanics. ${ }^{[1]}$ As we shall soon explain, we do not think this claim is well founded. Nevertheless, it is undeniable that the behavior of black holes in quantum mechanics presents conceptual challenges and opportunities that have not been adequately met. Before we describe the concrete new results in this paper, it seems appropriate to describe our view of the bigger picture to which they belong.

In fact the quantum mechanics of black holes presents two sets of problems, with different characters. They might be called the microscopic and the macroscopic problems. The microscopic problems concern the structure of very small black holes, with mass of order the Planck mass. It is difficult, for several reasons, to imagine that the description of such holes (if they exist) could fail to require a fully developed theory of quantum gravity.

The first and simplest reason is that as the mass of a black hole approaches the Planck mass, its Schwarzschild radius $2 G M$ approaches its Compton radius $\hbar / M$. Thus irreducible quantum fluctuations in position, which are of order the Compton radius, render the classical concept of the horizon - or indeed the classical concept of the gravitational field near the nominal location of the hole - problematic. In this regard it is instructive to consider briefly the behavior of ordinary elementary point particles on the other side of the dividing line, i.e. with mass much less than the Planck mass. The gravitational field of the point particle at the most naive level would be described by a Schwarzschild solution, with horizon at $R=2 G M=2 \hbar M / M_{\mathrm{Pl}}^{2}$. However when this $R$ is comparable to or less than the Compton radius $R_{\text {Compt. }}=\hbar / M$ the naive description is quite inappropriate - and we quite properly do not regard such elementary particles as black holes. Rather, to calculate the influence of the gravitational interaction between such a 
particle and another particle, we simply calculate Feynman graphs for graviton exchange. (Thought)-experimental attempts to "see" the nominal gravitational field at distance $R$, by considering scattering at very small impact parameter (and therefore large momentum and energy, of order $\hbar / R$ ), are doomed to failure. In such scattering, the amplitude for production of many pairs becomes large, and this situation simply cannot be described in terms of scattering from an external field. This behavior is of course drastically different from what one would have for a classical black hole, which simply absorbs particles incident at impact parameter less than the Schwarzschild radius. It is not at all clear how to interpolate between them.

Another difficulty is that as the mass of the hole decreases toward the Planck mass, the loop expansion parameter for the gravitational corrections to the effective Lagrangian approaches unity near the horizon, even if we imagine the ultraviolet divergences of this expansion are cut off. (See the discussion immediately below.) Thus the problem of understanding the non-perturbative behavior of quantum gravity, which includes its ultraviolet behavior as a sub-problem, cannot be avoided. At that point one must either throw up one's hands or (what in the current state of the art amounts to the same thing) appeal to string theory.

Neither of these difficulties arise for black holes whose mass is much greater than the Planck mass. For such holes, the Schwarzschild radius is much larger than the Compton radius, so that quantum uncertainty in position does not seriously interfere with the determination of the space-time geometry. Also, the curvature is small (relative to the inverse Planck length) near, and therefore of course external to, the horizon. In this case, a semiclassical treatment of gravity is quite plausible. To be more precise, if we assume that higher-order corrections to the effective action for gravity have an effective ultraviolet cut-off of order the Planck mass, then these corrections will be small near and external to the horizon. For example the contribution to the action from a potential correction term of the form $R_{\alpha \beta \gamma \delta} R^{\alpha \beta \gamma \delta}$ to the Lagrangian density, where $R^{\alpha \beta \gamma \delta}$ is the Riemann curvature tensor, will on dimensional grounds occur with a coefficient $M_{\mathrm{Pl}}^{-2}$. Therefore, since the only 
relevant scale at the horizon is set by the Schwarzschild radius, the contribution of this term to the equations of motion will be of order $\hbar^{2} /\left(R_{\text {Schwarzschild }}^{2} M_{\text {Pl. }}^{2}\right)=$ $\left(M_{\mathrm{Pl} .} / M\right)^{2}<<1$ relative to the contribution from the ordinary Einstein term near the horizon, and even smaller outside. Since the region inside the horizon is causally disconnected from the exterior of the black hole, the occurrence of truly large curvature near the singularity inside the horizon is not directly relevant to the physics seen by external observers, and the higher order corrections to the effective Lagrangian may be neglected everywhere in the physically relevant region. (Of course, this discussion, since it appeals to the classical description of the metric, is valid only semi-classically - regarding the geometry as approximately fixed - and to all orders in $\hbar$, but not necessarily beyond.)

In the macroscopic regime of large black holes, interesting effects can still arise from cumulative effect of small curvature over large volumes. In this regime, even in the absence of a workable complete theory of quantum gravity, one may hope to do semiclassical and perturbative calculations that have a high degree of plausibility and yet present interesting global features.

Indeed, striking phenomena have been found in this regime, notably the Hawking evaporation ${ }^{[2]}$ and the Bekenstein-Hawking entropy ${ }^{[2,3]}$ of black holes. The radiation, because of its approximately thermal nature, suggests that a stochastic element enters essentially into the description of macroscopic black holes. The most radical suggestion is that black hole evaporation allows in principle the evolution of pure into mixed states, which of course would violate the normal laws of quantum mechanics. For it seems clear that a black hole could be formed from the collapse of diffuse matter initially in a pure quantum-mechanical state, and if the subsequent radiation from the evaporating black hole were accurately thermal the evolved state would be mixed at later times. ${ }^{[1]}$ Strictly speaking it is not true that the radiation is accurately thermal, if for no other reason then because the mass, and therefore the nominal temperature, of the black hole changes with time. This is the simplest and most basic of all back-reaction effects, which correlate earlier with later radiation, but not the only one. Further correlations could in principle 
be calculated order-by-order in $\hbar$, but it is most unlikely that they are adequate to avoid the core of the problem - the apparent threat to normal quantum mechanics - posed by the stochastic radiation. The core of the problem is qualitative: for the causal structure characteristic of a classical black hole, the region at spatial infinity outside the black hole is not a complete (backwards) Cauchy surface; it does not allow one to uniquely connect the past and the future (See Figure 1 and its caption).

To pose the core problem in the clearest and most dramatic way, suppose the hole evaporates completely. Then the information about the wave function that flowed through the horizon seems to have disappeared permanently, and thus it appears that a pure state has evolved into a mixed one.

There are several possibilities to avoid this affront to quantum physics, which is perhaps the most challenging conceptual problem posed by macroscopic black hole quantum mechanics. We shall now briefly discuss four of them.

One possibility is that the radiation is sufficiently correlated to be a pure state all by itself, despite the fact that it appears almost totally uncorrelated (that is, the result of a slow thermal leak) in the standard semi-classical calculation. Now because the evaporation of large black holes is slow, the putative influence of earlier on later radiation must be reflected in some quasi-static property of the hole. That is, the hypothesis that the radiation all by itself contains a enough information to determine the state - that none is truly lost through the horizon - requires that the black hole should be capable of storing some accurate, stable record of how it was formed and what it has radiated. Until recently the conventional wisdom has been that this could not be true: that black holes have no hair, that is no (or very few) internal states. Indeed, it has been demonstrated fairly rigorously that at the classical level there are very limited possibilities for hair, ${ }^{[4]}$ and that each possibility requires the existence of suitable massless gauge fields. ${ }^{[5,6]}$ However, the major point of this paper will be to demonstrate that there are additional possibilities for hair, when the quantum nature of the black hole is taken into account. We certainly do 
not claim to have found enough hair to solve the main conceptual problem under discussion, but it is entirely possible that further analysis along these lines will uncover more.

A variant on this possibility arises if the black hole does not evaporate completely. Then it may leave a stable remnant whose internal state could be correlated with the state of the emitted radiation. This again requires that the stable remnant should be capable of supporting lots of hair. In this regard it is appropriate to recall that the classic stable black holes (extreme Kerr-Newmann holes) are calculated to have a very large entropy, proportional to the area of the event horizon. ${ }^{[7]}$ These calculations do not identify the quantum states which the entropy presumably is averaging over. These internal states represent another form of hair. Because the entropy is calculated to be proportional to the area of the event horizon, it is tempting to speculate that the internal states are associated with the state of the horizon, regarded as a quantum-mechanical object. ${ }^{[8]}$ However the exact nature of this hair, and its relation to the quantum hair we shall discuss in the bulk of this paper, is unclear to us at present.

A third possibility is that in some real sense there is no physical singularity. Physical behavior at arbitrarily large space-time curvature, such as formally appears near the black hole singularities, very plausibly brings in new degrees of freedom in addition to the ones familiar from our low-energy, low-curvature experience, which may drastically affect the nature of the singularity. This, in turn, can significantly change the nature of the conceptual problems in the quantum theory of black holes. Suppose, for example, that the singularity becomes timelike and naked - a possibility realized for a recently discovered class of black holes (extreme dilaton black holes with $a>1) .^{[9,10]}$ In the dilaton black holes there is a timelike singularity visible from infinity, and to define the quantum theory of fields in such a geometry appropriate boundary conditions must be imposed at the singularity. These boundary conditions are not uniquely fixed by the macroscopic theory, but would be determined by the underlying microscopic theory (e.g. superstring theory) needed to describe regions of truly large curvature. If the boundary 
conditions are correctly chosen, it is entirely reasonable to suppose that unitarity may be maintained. There is a well-known precedent for this situation, in the behavior of gauge theory magnetic monopoles (Callan-Rubakov effect). ${ }^{[1]}$ In that case, at the level of the effective field theory of electromagnetism, the monopole has a singularity at the origin. Spin- $\frac{1}{2}$ fermions with the minimal charge consistent with the Dirac quantization condition, viz. $e g=\frac{1}{2}$, feel no centrifugal barrier and in s-wave scattering reach the center with finite probability. Suitable boundary conditions can be supplied, so that the S-matrix describing this problem is unitary. One can also describe non-singular monopoles in a non-abelian extension of the low-energy model. Then the scattering problem is entirely well defined. Nevertheless, the description of low energy scattering in the extended theory can be accurately described by the effective low energy theory; the rich additional physics of the full theory, in this limited context merely serves to fix the boundary conditions. For space-like singularities such as those which occur for conventional black holes, however, boundary conditions would amount to constraints on the form of the wave function, which are presumably not physically sensible. (How does the initial wave function know it is going to describe collapse to a black hole, and had better acquire intricate non-local correlations?)

The only apparent problem with this possibility is that it requires rather special field content, and even so occurs only for extreme charged black holes. Thus it seems unlikely to be relevant to describe really large uncharged black holes, for which a self-consistent description in terms of low-mass fields outside the horizon ought to be a good approximation, unless there are low-mass fields (e.g. axions, dilatons) whose existence has for some reason eluded observation to date.

Finally, perhaps the most straightforward way out is to deny that information is truly lost down the singularity. As a model which is close to classical, and thus easy to discuss, suppose for example that the field content of the theory is somehow altered so that high curvature induces a rebound of the metric, so that the deep interior of the black hole rather than containing a singularity opens up into another nonsingular space - this will be the spore of a "baby universe." 
(Note that the singularity theorems, which say that singularities are inevitable, always assume constraints on the energy momentum tensor which are not true for generic forms of matter, and certainly need not apply to the effective Lagrangian at extreme curvatures.) This spore is necessarily causally disconnected from our own, at the classical level, because it is behind the horizon. Information about the wave function flowing through the horizon is now stored in the spore, rather than being lost at a singularity. It is then conceivable that the final act in the complete evaporation of the black hole involves the spore pinching off, to form a separate, self-contained baby universe. ${ }^{[12]}$ The baby universe may or may not resemble a fullscale universe with an interesting cosmology. Also the pinching off process may or may not be rare, depending on unknown details of the matter content - it is even conceivable that the typical black hole gives birth to many babies.

Do these possibilities help us avoid the evolution of pure into mixed states? At first sight it seems they give no help at all, but rather sharpen the problem. We have thrown information into the baby universe, where it is forever lost to us. In other words there are correlations between the state of the baby universe and the state of the parent universe, and therefore either alone is described by a mixed state. However, we must recognize that the strict separation of baby and parent is an approximation valid only at the classical level. If a baby universe can branch off quantum mechanically, it can also come back quantum mechanically. This simple but crucial remark implies that the proper description of the final state must include the state of the baby together with the parent; even if we inhabit the latter, we are not allowed to ignore the former. The penalty for this form of child neglect is revocation of license to practice quantum mechanics.

This discussion is closely related to the arguments made by one of us concerning the description of wormhole processes as effective interactions. ${ }^{[13-15]}$ While the wormhole process might be roughly considered as the birth of a baby universe, in a more careful discussion one must consider it as coherent emission into a "bath" of baby universes, a process whose macroscopic phenomenological consequences in our universe can be captured in an effective vertex, whose precise form depends on 
the wave function describing the bath. In this description, the difference between what the black hole absorbs and what it emits is correlated with the baby universe wave function, and quantum coherence is explicitly maintained.

Thus, to summarize, it does not seem that one is forced to conclude that the process of black hole evaporation violates or transcends the normal laws of quantum mechanics. There are several ways whereby one can imagine reconciling the stochastic element of black hole evaporation, and the apparently irreversible flow of information through the horizon, with the principles of quantum mechanics (and a unitary time evolution.) Some, though not all, of these possibilities require the existence of new, essentially quantum-mechanical, internal states - quantum hair - for black holes.

\subsection{Black holes AND ElEMENTARY PARTICle PROPERTIES}

Besides the fundamental challenge to the principles of quantum mechanics mentioned above, the known behavior of black holes creates a certain tension in the description of matter. In the standard semiclassical treatment of a black hole, the hole is described as a thermal object with very few internal degrees of freedom. The normal description of an elementary particle is very different - no temperature appears, and the particle may have many internal quantum numbers. Yet one might like to believe that there is no fundamental distinction between these forms of matter; that a sufficiently heavy elementary particle (any one heavier than the Planck mass) would in fact be a black hole.

In order to reconcile these descriptions, one necessary step is certainly to show that black holes are capable of carrying internal quantum numbers, contrary to the spirit of the classic no-hair theorems. That is what we shall accomplish below.

One more or less plausible possibility for black holes in the real world is precisely that there actually are elementary particles of this sort. For example, if electromagnetic gauge symmetry is first unified into a compact gauge group at an extremely large (super-Planckian) mass scale, then the stable magnetic monopoles 
will be black holes. These could be formed in the big bang, either directly or as the remnants or radiation products of the evaporation of other mini-black holes. Our considerations show that there are additional possibilities - that tiny black holes can be stabilized against Hawking evaporation by other gauge charges, associated with broken gauge groups. Thus if we are ever lucky enough to encounter a stable mini-black hole, the reason for its stability might not be at all obvious. And indeed there could well be a rich spectrum of such objects, with different masses, magnetic charges, and discrete charges.

\subsection{Summary OF the CONTENT}

The content of the rest of this paper is as follows. In Section 2 we discuss the reasoning leading to the classic (and classical) no-hair theorems of black hole physics. We then argue on physical grounds that these theorems must be violated in the case of discrete gauge charge. Concrete models embodying this physics, and the related physics of discrete magnetic charge, are exhibited. In Section 3 we discuss in careful detail the quantum treatment of the ordinary Reissner-Nordström black hole. The formal implementation of the charge projection is fully discussed, and shown to be accomplished by a weighted integral over field configurations with specified "vorticity" (line integral $\oint d \tau A_{\tau}$, where $\tau$ is the periodic imaginary time variable). The formal difference between electric and magnetic charge is emphasized. In Section 4 this apparatus is adapted to the case of discrete electric gauge charge. It is shown that the effect of discrete gauge charge is embodied in an imaginary time vortex configuration, and is non-perturbative in $\hbar$. It is argued that this configuration corresponds to the space-time process (discussed in Section 2) whereby a virtual cosmic string wraps around the black hole, and is in this sense to be interpreted as a world-sheet instanton for the cosmic string. Several charge-dependent physical observables, including non-vanishing electric fields outside the horizon and corrections to the classic relation between temperature and mass for the hole, are calculated in appropriate limits. In Section 5 we discuss the corresponding physics for discrete magnetic charge. It is argued that although 
there are drastic formal differences between electric and magnetic charge, it is quite plausible that their physical behavior is not drastically different. In Section 6 we discuss the dual description of broken symmetry phases. This material has nothing to do with black holes per se, but the material is not entirely standard and is used in the following section. One simple but striking result is that in the dual description of the Higgs phase, where the phase of the scalar field is represented in terms of a rank-two antisymmetric tensor field $B$, the charge coupling takes the form of an interaction $B \wedge F$ that resembles a $\theta$-term. In Section 7 we discuss an alternative approach to discrete gauge hair more along the lines presented in the literature as "axion charge", and attempt to clarify the relationship between these approaches. Finally in Section 8 we draw the distinction between primary and secondary quantum hair. We give examples of secondary hair within the standard model of particle physics, and emphasize that the discrete hair discussed in the bulk of the paper is primary.

We shall adopt units in which $c=1$, but will display factors of $G$ and $\hbar$.

\section{Discrete Gauge Hair: Preliminary Discussion}

In this chapter we shall review the conceptual framework for no-hair theorems, and then discuss why these theorems are not expected to apply in the case of discrete gauge hair. Several of the topics discussed here in physical terms will be discussed more formally in the following chapters.

\subsection{NO-HAIR THEOREMS}

To understand the essence of the no-hair theorems, ${ }^{[16]}$ it is best to begin by considering the simplest case of a scalar field in the background of a Schwarzschild black hole. The metric, in Schwarzschild coordinates, is

$$
d s^{2}=\left(1-\frac{2 G M}{r}\right) d t^{2}-\left(1-\frac{2 G M}{r}\right)^{-1} d r^{2}-r^{2} d^{2} \Omega
$$

where, of course, $d^{2} \Omega$ is the line element on the unit sphere. In analyzing the wave 
equation in this background, it is convenient to introduce the "tortoise coordinate"

$$
r_{*} \equiv r+2 G M \ln [(r-2 G M) / 2 G M]
$$

which satisfies

$$
d r_{*}=\left(1-\frac{2 G M}{r}\right)^{-1} d r
$$

Because the metric at fixed angle is proportional, in this variable, to that of flat space:

$$
d s_{\text {fixedangle }}^{2}=\left(1-\frac{2 G M}{r}\right)\left(d t^{2}-d r_{*}^{2}\right)
$$

the wave equation will assume a particularly simple form.

It is crucial to note that $r_{*}$ has the properties

$$
\begin{aligned}
r_{*} \rightarrow r+2 G M \ln r ; & r \rightarrow \infty \\
r_{*} \rightarrow \ln (r-2 G M) \rightarrow-\infty ; & r \rightarrow 2 G M .
\end{aligned}
$$

The first of these equations implies that $r_{*}$ reduces essentially to $r$, and in particular that it becomes positively infinite, as $r \rightarrow \infty$. (The extra logarithm here is due to the long-range nature of the gravitational interaction, and directly reflects the "Coulomb logarithm" in the phase of waves at infinity.) More important for our present considerations is the second equation. It tells us that $r_{*}$, the natural variable for the wave equation, approaches negative infinity at the horizon. In this sense, the horizon is "infinitely far away."

The wave equation is easiest to analyze by inserting the partial wave expansion for the scalar field $\Psi$

$$
\Psi(t, r, \Omega)=\frac{1}{r} \sum_{l, m} \psi_{l, m}\left(t, r_{*}\right) Y_{l m}(\Omega)
$$

into the wave equation. One obtains

$$
\left(-\frac{\partial^{2}}{\partial t^{2}}+\frac{\partial^{2}}{\partial r_{*}^{2}}\right) \psi_{l, m}=\left(1-\frac{2 G M}{r}\right)\left(\mu^{2}+\frac{2 G M}{r^{3}}+\frac{l(l+1)}{r^{2}}\right) \psi_{l, m}
$$

where $\mu$ is the mass (really the inverse Compton wavelength) of the scalar. The 
right-hand side of this equation may be interpreted as an effective potential. This effective potential vanishes near the horizon (i.e. as $\left.r_{*} \rightarrow-\infty\right)$, approaches simply $\mu^{2}$ as $r_{*} \rightarrow \infty$, and is everywhere positive.

Now consider the zero-frequency limit. Clearly, because the second derivative never changes sign, a solution that decreases exponentially at infinity will have to blow up at $r_{*} \rightarrow-\infty$, i.e. at the horizon. Such behavior is physically unacceptable: it involves infinite energy in the $\Psi$ field, among other things. Thus, there are no physically acceptable static solutions; the only acceptable static scalar field configuration is identically zero. ${ }^{[5]}$ In particular there can be no exponentially falling Yukawa tails, as would occur far from a normal point source coupled to $\Psi$. A black hole cannot be a source: it has no hair.

The crucial circumstance underlying this result is clearly the fact that the natural variable $r_{*}$ is unbounded in both directions, approaching negative infinity at the horizon. It is for this reason that continuation of any possible tail from spatial infinity costs not merely a large, but inevitably an infinite, amount of energy.

Price $^{[16]}$ analyzed wave equations for higher integer spin fields as well. Unfortunately these equations are most conveniently analyzed using the NewmanPenrose $^{[17]}$ formalism, which may unfamiliar to many readers. Furthermore the equations, although satisfactory in flat space, are inconsistent in curved space for spin $s \geq 3$. Thus there is more than one large hole appearing in the analysis. However since the question of higher-spin hair is potentially very important, we will now briefly summarize and make some tentative observations on the analysis.

Using the Newman-Penrose formalism, and extending the flat-space equations formally by minimal coupling, one arrives at equations of the form

$$
\frac{d^{2}}{d r_{*}^{2}}\left(r^{s+1} \hat{\Phi}_{0}\right)-F_{l}\left(r_{*}\right) r^{s+1} \hat{\Phi}_{0}=0
$$

for the static part of the $l^{\text {th }}$ partial wave of the fundamental quantity $\hat{\Phi}_{0}$. Actually $\hat{\Phi}_{0}$ is the coefficient of a spherical harmonic $Y_{l m}$, and there are $2 l+1$ independent 
components, which we shall leave implicit. (Eq. (2.8) is derived by simple manipulation of two particular components of the minimally coupled Newman-Penrose system of equations. If we had chosen other components and done more complicated manipulations, we would have gotten a different equation for $\hat{\Phi}_{0}$ - this is the inconsistency mentioned above. However any "reasonable" manipulation leads to an equation of the same general form as (2.8), but with a modestly modified function $F_{l}$.) Here

$$
F_{l} \equiv(1-2 G M / r) \frac{l(l+1)}{r^{2}}
$$

$\hat{\Phi}_{0}$ is the de-spun field of zero conformal and spin weight. The two crucial properties of $\hat{\Phi}_{0}$ are that:

1. The ordinary spinor or tensor components of the fields may be obtained from $\hat{\Phi}_{0}$ by differentiation and algebraic manipulations.

2. $\hat{\Phi}_{0}$ is directly physically meaningful, and is expected to be well-behaved both at the horizon and at infinity. For example in the electromagnetic case $s=1$ one finds $\hat{\Phi}_{0}=-\frac{1}{2}\left(E_{r}+i B_{r}\right)$. Because the field strengths (and not the potentials) occur here, $\hat{\Phi}_{0}$ is physically meaningful. Furthermore because the radial components of the field strength are unaffected by boosts in the radial direction, the physical requirement that freely falling observers see no singular behavior at the horizon entails that $\hat{\Phi}_{0}$ must be non-singular at the horizon. Of course, manifestly it must be well-behaved at infinity.

Taking eq. (2.8) at face value, one sees that there is a fundamental distinction between $l \leq s-1$ partial waves and the higher ones. In both cases one has the asymptotics $\hat{\Phi}_{0} \rightarrow a_{l}+b_{l} r_{*}$ for solutions near the horizon $r_{*} \rightarrow-\infty$, with only $b_{l}=0$ being physically acceptable. As $r_{*} \rightarrow \infty$ the solutions behave as $\hat{\Phi}_{0} \rightarrow c_{l} r_{*}^{l-s}+d_{l} r_{*}^{-l-s-1}$, and here there is a big difference. For $l \geq s$, this solution is physically acceptable only if $c_{l}=0$. But one may repeat the argument given for the case of scalar fields to show that the solution that is nonsingular at the event horizon $\left(a_{l} \neq 0, b_{l}=0\right)$ matches up with a solution with $c_{l} \neq 0$ at $r_{*}=\infty$. 
Therefore, the only allowed static solution has $\hat{\Phi}_{0}$ identically zero - there is no hair. However, for the lower partial waves, this argument does not apply, and acceptable non-trivial solutions do indeed exist.

The characteristic feature of these allowed solutions is that the field carries a non-vanishing value of a conserved surface integral. Propagation of radiation to spatial infinity cannot change the value of this surface integral. Since no causal process, including in particular the formation of a black hole, can flout this conservation law, one expects that the conserved surface integral corresponds to a variety of hair that can reside on a stationary black hole.

As we have mentioned, the full set of equations for higher spin fields in curved space is inconsistent. However, as Price argues, it is quite plausible that the (unknown) corrections that lead to consistent equations, while they will alter the form of the effective potential and lead to mixing among modes of the same quantum numbers, will not alter the crucial asymptotic behaviors near the horizon and spatial infinity. This is because at spatial infinity one should have something arbitrarily close to the flat space equations, while close to the horizon the effective potential is always killed by the $1-2 G M / r$ stretching factor.

When mass terms are introduced into the field equations, the analysis of classical hair on stationary black holes is qualitatively altered. This can be discussed most cogently in the spin-1 case, where no issue of consistency arises. For a massive spin-1 field, there is no conserved surface integral, and hence no compelling reason for spin-1 hair (that is, electric or magnetic charge) to reside on a black hole. Indeed, the analysis of the field equations shows that the physically acceptable solution at the horizon fails to match up with a decaying exponentially at infinity, so that only the trivial solution is allowed. ${ }^{[5,6]}$ It is instructive to consider what happens, in the case of a vector field with mass $\mu$, in the limit $\mu \rightarrow 0$. When a charged particle that acts as a source for a massive vector field falls through the horizon of a black hole, the vector field outside the horizon leaks away in a (Schwarzschild) time of order $\mu^{-1}$. Thus, as $\mu$ gets smaller, the time scale for the decay of the field 
gets longer and longer; finally, in the limit $\mu \rightarrow 0$, the field persists indefinitely.

Apart from the question of consistency, at the level of formal manipulation and asymptotic behavior the Newman-Penrose-Price equations for higher spins do not look very different in principle from those for spin 1. In the body of this paper we shall analyze spin 1 in depth, and show that soft mass terms (arising from spontaneous breakdown of the gauge invariance associated with massless spin1 fields) do not destroy the hair entirely, if there is a remnant discrete gauge symmetry left behind. In many ways the crucial ingredient of the argument is the construction of appropriate charge projections; and this part could be carried through for higher spin too. However the physical interpretation of the hair only emerges clearly when one can implement a Higgs mechanism and construct vortices; this might be doable for higher spins at least in flat space, and presents an attractive subject for further investigation.

A simple but perhaps not entirely misguided way to regard the no-hair theorems is as follows: in collapse to a black hole, any information that can fall through the horizon will fall through the horizon. The universal action of gravitation sweeps clean; and all trace of what has been swept in disappears, because once beyond the horizon it loses causal contact with the exterior. The only quantities that escape destruction are those which can be seen from the safe distance of infinity; that is, those connected with conserved surface integrals, such as the ones we have discussed above.

\subsection{Discrete Gauge Symmetry}

Upon first hearing, the notion of local discrete symmetry in the continuum may sound rather silly. Indeed, the most important dynamical consequence of a continuous local symmetry is the existence of a new field, the gauge field. This field arises when one introduces a gauge potential, in order to formulate covariant derivatives. Covariant derivatives are of course necessary, so that invariant interactions involving gradients may be formed. Such interactions in turn are necessary in 
order that charged fields may propagate. In the case of a discrete symmetry there is no similar need to introduce a gauge potential, because the ordinary derivative already transforms simply.

Alternatively, since any path can be continuously deformed to a trivial path, the corresponding parallel transport can be continuously deformed to the identity. But for a discrete group this means we must have the identity all along: thus all parallel transport is trivial. Also, if the discrete gauge group arises as the remnant of an initially continuous group, after all continuous symmetries have been wiped out by the Higgs mechanism, one is accustomed to think that charge is completely screened.

Upon further reflection, however, one realizes that each of these arguments has serious limitations. First, there are situations in which one cannot deform all paths to the identity. This occurs, by definition, on spaces that are not simply connected. Second, there are situations in which the parallel transport is not a continuous function of the path, or is ill-defined for some paths. An important class of examples, which will be extensively considered below, concerns flux tubes in the Higgs phase of gauge theories. In that case the gauge symmetry "expands" to the full unbroken symmetry at the core of the flux tube. Parallel transport through this core will not in general stay within the discrete subgroup, so from the point of view of the low-energy theory (which does not take account of the expanded gauge symmetry) there is a singularity.

Likewise, the statement that in a Higgs phase charge is completely screened is too broad. One must appreciate that the screening occurring in the Higgs mechanism is not a mystical process, but essentially a special case of the homely phenomenon of dielectric polarization as occurs in ordinary dielectrics or plasmas. It is true that we are concerned, in the Higgs mechanism, with a particularly effective screening, produced by a condensate of massless charged particles. Such particles are capable of producing a dipole distribution, to exactly cancel any imposed electric field. The particles in the condensate transform trivially under the remnant 
discrete symmetry, however, and it is unreasonable to expect that any distribution of them can perfectly mimic a non-trivial source.

There is a profound difference between local and global symmetries, whether continuous or discrete. While global symmetry is a statement that the laws of physics take the same form when expressed in terms of various distinct variables, local symmetry is a statement that the variables used in a physical theory are redundant. In language that may be more familiar, this redundancy is often stated as the fact that in a gauge theory, only gauge invariant quantities are physically meaningful. From this point of view, it is clear that no processes, not even such exotic ones as collapse to a black hole or black hole evaporation, can violate a gauge symmetry. On these very general grounds, then, we should expect that a black hole must be capable of carrying discrete gauge charges, reflecting the charges of what fell in to make it. However, these abstract arguments leave it totally unclear what the concrete physical implications of discrete gauge charges could be.

\subsection{Discrete Electric GAuge HAIR}

To address this question, and to make the whole discussion more concrete, let us now consider a specific realization of the general idea of discrete local symmetry. ${ }^{[18]}$ Consider a $\mathrm{U}(1)$ gauge theory containing two complex scalar fields $\eta, \xi$ carrying charge $N e, e$ respectively. Thus we have for the Lagrangian

$$
\left.\mathcal{L}=-\frac{1}{4} F_{\mu \nu} F^{\mu \nu}+\left|\left(\partial_{\mu}+i N e A_{\mu}\right) \eta\right|^{2}+\mid\left(\partial_{\mu}+i e A_{\mu}\right) \xi\right)\left.\right|^{2}-V(|\eta|,|\xi|)
$$

Suppose that $\eta$ undergoes condensation at some very high mass scale $v$, while $\xi$ produces quanta of relatively small mass and does not condense.

Before the condensation the theory is invariant under the local gauge transformations

$$
\begin{gathered}
\eta(x)^{\prime}=\exp (i N e \Lambda(x)) \eta(x) \\
\xi(x)^{\prime}=\exp (i e \Lambda(x)) \xi(x) \\
A_{\mu}(x)^{\prime}=A_{\mu}(x)-\partial_{\mu} \Lambda(x)
\end{gathered}
$$


where $\Lambda$ is a real variable. However the condensate characterized by the vacuum expectation value

$$
<\eta(x)>=v
$$

in the homogeneous ground state is invariant only when $\Lambda$ is an integer multiple of $2 \pi / N e$. These residual transformations still act non-trivially on $\xi$, multiplying it by various $N^{\text {th }}$ roots of unity. Their possible actions generate the discrete group $Z_{N}$.

The effective low energy theory well below $v$ will simply be the theory of the single complex scalar field $\xi$; neither the gauge field nor $\eta$ will appear in the effective theory, since these fields produce very massive quanta. The only implication of the original gauge symmetry for the low energy effective theory is then the absence of interaction terms forbidden by the residual discrete symmetry. (If there were additional charged scalar fields in the theory, the discrete symmetry would forbid many couplings that were otherwise possible.) But this implication, so far, does not distinguish between local and global symmetry.

Once we widen our horizons to consider processes occurring at energies of order $v$, of course the underlying gauge degrees of freedom, if they exist, can be excited. A more subtle manifestation is also possible. The broken symmetry theory contains stable strings threaded by magnetic flux $2 \pi / N e$, as follows. The theory supports vortex solutions, where the $\eta$ field behaves asymptotically as a function of the angle $\theta$ as

$$
\eta(r, \theta) \rightarrow v e^{-i \theta}, r \rightarrow \infty
$$

where $v$ is the magnitude of $\eta$ in the homogeneous ground state. Along with this asymptotic behavior for $\eta$ we must have for the gauge potential

$$
A_{\theta}(r, \theta) \rightarrow \frac{1}{N e}
$$

so that the covariant derivative $D_{\theta} \eta=\left(\partial_{\theta}+i N e A_{\theta}\right) \eta$, which appears (squared) in the energy density, vanishes at infinity. 
In this set-up the field strength also vanishes asymptotically. Indeed we note that by making the choice $\Lambda=\theta / N e$, we can formally remove the space dependence of $\eta$ in (2.13) and make $A_{\theta}$ in (2.14) vanish altogether. In so doing, we have (formally) transformed back to the homogeneous ground state. However of course the gauge transformation function $\Lambda$ is not quite kosher, since the angle $\theta$ is not a legitimate single-valued function. The correct statement is that the asymptotic behavior of the vortex is trivial and can be gauged away locally, but not globally. Since we can pick a well-defined branch of $\theta$ in any patch that does not surround the origin, all local gauge invariant quantities must reduce to their ground state values - this explains, if you like, why $D \eta$ and $F$ vanish. But the line integral of $A$ around a closed loop surrounding the origin, which according to Stokes measures the flux inside, cannot be changed by any legitimate gauge transformation, and it is definitely not zero for the vortex. Indeed we find the basic flux unit is $\Phi_{N}=2 \pi / N e$.

Another perspective on the global non-triviality of the vortex, is that our putative gauge transformation $\Lambda=\theta / N e$ transforms a unit charge field such as $\xi$ into something that is not single-valued: following (2.10) we find that $\xi^{\prime}(\theta+2 \pi)=\exp \left(\frac{2 \pi i}{N}\right) \xi^{\prime}(\theta)$.

What has all this got to do with discrete gauge theories in the continuum? Well, the condensate $\langle\eta\rangle=v$ is not invariant under a general gauge transformation, but it is invariant under the discrete subgroup generated by $\Lambda=2 \pi / N e$. This discrete subgroup acts trivially both on $\eta$ and, it would seem, on the gauge field ( $\Lambda$, since it takes only discrete values, cannot change continuously at all). However, $\Lambda$ and its various powers are definitely not trivial acting on $\xi$, which gets multiplied by powers of the $N^{\text {th }}$ root of unity. Thus there is a discrete but non-trivial gauge subgroup left. Moreover, the gauge transformation associated with winding around a vortex is precisely an element of the residual discrete gauge symmetry group this is just a restatement, in our new interpretation, of the result of the previous paragraph. Alternatively we could say that the Wilson loop for parallel transport 
around the vortex defines an element of the residual gauge group:

$$
\exp \left(\oint i e A_{\mu} d x^{\mu}\right) \in Z_{N}
$$

We anticipated that the discrete gauge group might come into its own in the presence of singularities. Now we see how this is quite simply realized. The required "singularity" is here supplied by the vortex core. In that core, where the condensate vanishes, the discrete gauge symmetry blows up into a full scale continuous $U(1)$, and the vector potential is unfrozen. The only trace of the vortex visible outside the vortex core is the total flux - which is none other than an element of the residual discrete gauge symmetry group.

The scattering of $\xi$ quanta, with charge $e$, from such strings ${ }^{[19]}$ is dominated, at low energies, by the Aharonov-Bohm effect. ${ }^{[20]}$ The magnitude and form of this cross-section is uniquely determined by the product of charge and flux, modulo $2 \pi \hbar$, and thus allows one, in principle, to make a precise observational determination of the $Z_{N}$-valued charge alluded to above. Of course postulating the existence of such strings takes us outside the framework of the effective low-energy theory as usually understood, so the existence of this effect does not really contradict the statement made above, that the low-energy effective theory does not distinguish local from global discrete symmetries.

A simple thought experiment ${ }^{[18]}$ based on the Aharonov-Bohm scattering process provides a heuristic but convincing demonstration that black holes have discrete gauge hair. For let us imagine that we have a $\xi$ quantum falling into a black hole, and scatter a string of very low energy and momentum from this composite object. The scattering cross-section, which involves behaviors at large times and distances, should not depend on the precise instant at which the particle crosses the event horizon - a rather fuzzy notion, in any case. And yet this cross-section does depend critically on the $Z_{N}$ charge. We must conclude that this $Z_{N}$ charge

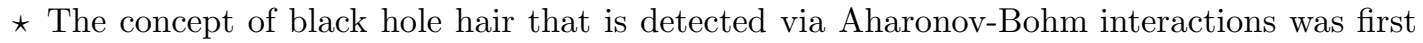
discussed in Ref. 21.
} 
does not depend on whether the particle has crossed the event horizon, and in particular that it retains its meaning (and induces the same Aharonov-Bohm phases) for the asymptotic, "pure" black hole. A more formal proof, whose core idea is really the same, could be based on the discrete analog of Gauss' law, which insures that the $Z_{N}$ charge inside a volume can be expressed in terms of the expectation values of quantum field operators on the bounding surface. ${ }^{[22,23]}$

We would now like to discuss why this discrete electric gauge hair is properly quantum hair, with no ordinary classical limit. In a sense this is obvious from the nature of screening. In a Higgs theory, there is a condensate of massless charged particles in the ground state, and at the classical level this condensate is ready and willing to screen any test charge. The only thing that prevents perfect screening is the quantization of the charge of the condensate particles. Thus, the survival of any consequences of discrete electric gauge hair must depend on quantization. To better appreciate this, it is helpful to understand more explicitly how the charge units vary with $\hbar$. The classical action, obtained by integrating the Lagrangian density (2.10) over space and time, must have the units of $\hbar$. From this it follows easily that the $e$ appearing in the Lagrangian has units of (action) ${ }^{-\frac{1}{2}}$. For clarity we shall write this quantity as $e_{f}$, ( $f$ for field charge). In the ordinary classical limit, $e_{f}$ is held fixed. On the other hand the charge density operator of the theory, which multiplies $A_{0}$ in the Lagrangian, is of the generic form $i e_{f} \phi^{\dagger} \overleftrightarrow{\partial}_{0} \phi$. Because the fundamental commutation relation normalizes a bilinear form in $\phi$ and $\partial_{0} \phi$ to $\hbar$, it follows that the charge operator is essentially $\hbar e_{f}$ times the number operator. Thus the charge $e_{p}$ ( $p$ for particle) of a single quantum is

$$
e_{p}=\hbar e_{f}
$$

We see that electric charges which are only finite multiples of $e_{p}$ vanish in the ordinary classical limit. (It will be useful to remember that $\hbar e_{f}^{2}$ and $e_{p}^{2} / \hbar$ are dimensionless quantities.)

It would be both disturbing and disappointing if the only manifestation of discrete quantum hair on a black hole involved explicit cosmic strings. Among 
other things, this would close off some of the ideas mentioned in the introductory chapter, which require hair capable of influencing ordinary particles. And indeed if one has a real process sensitive to the charge, it seems quite reasonable to expect that there are associated virtual processes also sensitive to the charge. Thus we might expect that the discrete charge manifests itself as an instruction concerning how to weight the relative phase of amplitudes for the black hole wrapping one way or the other around an appropriate cosmic string (or, of course, the string wrapping around the hole). Upon changing real to virtual, we expect that the discrete electric charge on the hole should instruct us how to weight the phase of amplitudes for processes where virtual cosmic string loops nucleate, envelop the hole, and re-annihilate. Described in other words, the relevant processes are ones in which virtual string world sheets wrap around the hole. ${ }^{[24-26]}$

In the following chapters, we shall verify this expectation in great detail.

The virtual string world sheet process is of course a tunneling process, and will be non-perturbative in $\hbar$; indeed it will be exponentially small in $1 / \hbar$. (Note that cosmic strings are classical objects: their structure follows from solving the classical field equations, and so their flux and size remain fixed as $\hbar \rightarrow 0$ with $e_{f}$ and $v$ fixed.) This contrasts with what one might expect for an ordinary particle carrying discrete charge, where there are small charge-dependent effects (say in the scattering of two charged particles) of order $e_{p}^{2}$ from the short-range Yukawa fields. The effect for black holes is different from, and much more subtle than, restoration of their lost Yukawa tails. Another important difference is that for black holes, but not for ordinary particles, discrete hair expands the space of states - see chapter 8. 


\subsection{Discrete Magnetic Hair}

Now let us discuss the magnetic analog of discrete electric gauge hair. At the most naive level one might expect that these are trivially related, because of the well-known duality property of the free Maxwell equations. And indeed in the context of classical black hole physics, the difference between electrically and magnetically charged holes is entirely trivial - both are described by essentially the same Reissner-Nordström, or Kerr-Newman, solutions. However the formal treatment of these objects in the semiclassical limit must be quite different, as we shall see in the next chapter. One circumstance that makes this plausible is that the electric charge quantum is $e_{p}=\hbar e_{f}$ while the magnetic charge quantum is simply $e_{f}^{-1}$, so the latter but not the former is finite in the classical limit. Nevertheless, at the end of the day the properties, specifically the radius and temperature, of an electric hole whose charge is many quanta are closely similar to those of the magnetic hole with the same magnitude of field strength and mass.

What about broken symmetry phases? If a gauge theory is in a weak coupling Higgs phase, electric fields are screened, and magnetic fields are confined to flux tubes. These phenomena can be described classically - the screening length and flux quantum are independent of $\hbar$. If a gauge theory is in a confinement phase, magnetic fields are screened, and electric fields are confined to flux tubes. These phenomena are quantum-mechanical; in fact, the inverse screening length is nonperturbative in $\hbar$.

We have seen that $Z_{N}$ electric charges can be introduced into a Higgs theory, such that the charges have an Aharonov-Bohm interaction with a magnetic flux tube. The construction of weakly coupled models with $Z_{N}$ magnetic monopoles that can be studied with semiclassical methods is a bit more involved. (There is an enormous literature concerned with discrete gauge theory monopoles at strong coupling, which are often invoked as an explanation of confinement; but as far as we know these ideas have never been pursued very far within the framework of a well controlled approximation.) 
Probably the simplest model, at least conceptually, is based on the spontaneous breakdown of the gauge group $S O\left(N^{2}-1\right)$ to $S U(N) / Z_{N}$. This may be accomplished by a Higgs field in the three-index antisymmetric tensor representation, $H_{i j k}$, that acquires a vacuum expectation value

$$
<H_{i j k}>=v f_{i j k}
$$

proportional to the structure constants $f_{i j k}$ of $S U(N)$. Note that $S U(N)$ is of dimension $N^{2}-1$. It may be regarded as a subgroup of $S O\left(N^{2}-1\right)$, because it can be represented as the group of orthogonal transformations (leaving invariant the trace of Casimir operator, or the Killing form) acting by conjugation on its Lie algebra. The condition that such a transformation is an isomorphism of the Lie algebra of $S U(N)$ is precisely that it leaves the structure constants invariant. The group of automorphisms of the Lie algebra of $S U(N)$ is $S U(N) / Z_{N}$, acting by conjugation. (The central elements, of course, do not generate non-trivial automorphisms this way.) Thus we see that $S U(N) / Z_{N}$ is nicely embedded in $S O\left(N^{2}-1\right)$, and that there is a simple way of breaking the larger group down to the smaller one. ${ }^{[27]}$

In this framework we may identify configurations carrying $Z_{N}$ magnetic flux, as follows. The homogeneous ground state defined by (2.17) is not unique; one may obtain states that are equally good energetically by acting on it with any $S O\left(N^{2}-\right.$ 1) transformation. Of course in this procedure the $S U(N) / Z_{N}$ is powerless, and the manifold of formally unequal (but gauge equivalent) candidate ground states is the coset space $S O\left(N^{2}-1\right) /\left(S U(N) / Z_{N}\right)$. Now let us suppose, as usual in constructing gauge theory monopoles, ${ }^{[28]}$ that the symmetry may break to different points within this manifold, depending on the direction in space, at spatial infinity. Now consider the parallel transport around a sequence of loops, each starting and ending at the north pole; the loop begins as an infinitesimal loop, expands to one which is a great circle through the south pole, and then comes back to another small loop, having lassoed the sphere once. Since each of these loops starts and ends at the north pole, the total parallel transport defined by each must leave the Higgs field at the north 
pole invariant; thus it must define an element of $S U(N) / Z_{N}$. Furthermore the small loops at the beginning and end must give the identity element in $S U(N) / Z_{N}$. Thus the sequence of parallel transports, as the loops lasso the sphere, defines an element of the homotopy group $\pi_{1}\left(S U(N) / Z_{N}\right)=Z_{N}$. The different homotopy classes cannot be connected to one another by continuous changes over a finite volume. Thus there is infinite energy barrier between them, and, minimizing the energy within each sector, one will find some stable monopole configurations. (Of course the doubly charged pole may in principle decay into two singly-charged poles, and so forth.)

Alternatively, the flux may be defined by patching hemispheres. On a hemisphere, one may perform an $S O\left(N^{2}-1\right)$ gauge transformation to rotate the vacuum expectation values so that they are all pointing in the same direction. Likewise, one may align the other hemisphere. However matching the gauge transformations along the intersection of these hemispheres will introduce a closed path in $S U(N) / Z_{N}$, as before.

The $Z_{N}$ magnetic charge, then, can be defined by essentially classical operations at infinity. In the black hole sector too, one should be able to find classical solutions with the specified asymptotic flux. (And later, we will.)

Thus we see that there is a magnetic analogue of discrete electric hair, though superficially it appears to differ from it qualitatively. However, on closer consideration one finds that the difference is not entirely sharp. Suppose, for example (not unrealistically), that the $S U(N) / Z_{N}$ theory we have been discussing so far classically, actually confines electric charge (and screens magnetic charge) at large distances. Then strictly speaking there will not be any magnetic flux at infinity. It might seem, then, that the magnetic black hole has been shaved bald. However in the confined phase one has electric flux tubes, analogous to the magnetic flux tubes of the Higgs phase, because now it is electric flux that is confined. These flux tubes will be able to detect the screened discrete magnetic charge on the black hole, by the dual of the Aharonov-Bohm process, scattering off electric flux tubes. ${ }^{[23,13,30]}$ 
This physics, of course, is entirely analogous to what we have seen in the Higgs phase.

(To avoid misunderstanding, we should probably emphasize that such screened magnetic hair does not occur in the standard model, with unbroken gauge group $\left[S U(3)_{\text {color }} \times U(1)_{\mathrm{em}}\right] / Z_{3}$. Though this model admits magnetic monopoles that carry a $Z_{3}$ color magnetic flux, there are no stable electric flux tubes - the tube can break via nucleation of a quark-antiquark pair. Hence there is no means of detecting $Z_{3}$ magnetic charge at long range. Furthermore, and not coincidentally, the $Z_{3}$ color magnetic charge of a monopole is completely determined by its $U(1)_{\mathrm{em}}$ magnetic charge; it is not an independent quantum number. If black holes can carry screened magnetic hair in Nature, this hair is not associated with the known strong interaction. Rather, it must be associated with another, as yet unknown, confining gauge interaction that admits genuine $Z_{N}$ monopoles.)

The real difference between the two cases is not qualitative, but only quantitative. Confinement is non-perturbative in $\hbar$, so that classically a magnetic charge generates magnetic flux at infinity; in a straightforward weak-coupling analysis, there is visible hair. Subtleties arise only after one realizes that there is an important effect at large distances, confinement, which is non-perturbative in $\hbar$. For if one then attempts to visualize this effect classically, or to incorporate it into an improved effective Lagrangian which one then treats classically, the classic (classical) no-hair argument will come into play. However, fortunately, the more straightforward electric screening case has alerted us that the hair remains, because of another nonperturbative effect in $\hbar$, namely the wrapping of real or virtual flux tubes around the hole. Thus if confinement is regarded as a strong effect, rather than as a tiny correction, the magnetic charge case comes to look very similar to the electric charge case. 


\section{The Reissner-Nordström Black Hole}

\subsection{The Euclidean Path Integral}

One of our objectives is to extend the standard semiclassical analysis of black hole thermodynamics to include effects that are nonperturbative in $\hbar$. Nonperturbative effects are most conveniently studied using Euclidean path integral methods, and we will use such methods here. In fact, we do not know another way to obtain our main results.

We employ the Euclidean path integral with reservations, because in the context of black hole physics, the foundations underlying this formalism are not completely secure. There are both technical and conceptual problems. The main technical problem is that the Euclidean Einstein-Hilbert action is unbounded from below. The integral over the conformal degree of freedom of the metric must be defined with some care, and it is not clear what the correct prescription is. The main conceptual problem arises because the Euclidean formalism, as we use it, applies to a black hole in thermal equilibrium with a surrounding radiation bath, rather than a black hole evaporating into empty space. The equilibrium is typically unstable if the radiation bath is infinite in extent, because the black hole has negative heat capacity. This difficulty can be avoided by enclosing the radiation in a sufficiently small reflecting cavity. The deeper question is whether the concept of a black hole in equilibrium with radiation makes sense. A stationary spacetime filled with radiation does not satisfy the Einstein equations. The back reaction of the radiation on the geometry causes the spacetime to evolve, so that it becomes unclear how the concept of thermal equilibrium can apply. (There is, of course, an approximate notion of thermal equilibrium in the semiclassical limit, since the temperature of the radiation is of order $\hbar$.)

We will ignore these problems in this paper. Our working hypothesis is that the notion of black hole thermodynamics, and the use of the Euclidean path integral to probe the thermal behavior, are sensible, at least in a semiclassical approximation. 
In this section, we will illustrate the Euclidean method by applying it to the case of a black hole that carries electric or magnetic charge (the ReissnerNordström black hole). We particularly want to emphasize how charge projection operators are inserted into the partition function, for the purpose of studying the thermodynamics of a particular charge sector. Similar charge projections will be invoked in the subsequent two sections, which treat the cases of screened electric and magnetic charge.

\subsection{The Semiclassical Limit}

We are interested in the thermodynamic behavior of a black hole in the semiclassical limit, the limit of small $\hbar$. To define this limit precisely, we must specify what quantities are to be held fixed as $\hbar$ approaches zero.

In a model of electromagnetism coupled to gravity, there are two coupling constants, Newton's constant $G$ and the electromagnetic gauge coupling $e$. Both of these are to be regarded as classical quantities; that is, they are held fixed as $\hbar \rightarrow 0$. We emphasize (again) that $e$ denotes here the coupling that appears in the classical action, and has the dimensions of $(\text { action })^{-1 / 2}$. Thus, $e$ is related to the electric charge $e_{\text {particle }}$ of an elementary particle by

$$
e_{\text {particle }}=\hbar e \text {. }
$$

The semiclassical limit of a black hole with mass $M$ and electric charge $Q$ is defined by holding both $M$ and $Q$ fixed as $\hbar \rightarrow 0$. Thus, in this limit, the mass becomes arbitrarily large compared to the Planck mass $(\hbar / G)^{1 / 2}$, and the charge becomes arbitrarily large compared to the charge quantum $\hbar e$. In terms of black hole thermodynamics, the length scale $\beta \hbar$ is held fixed, where $\beta^{-1}$ is the black hole temperature. In the semiclassical limit, then, this length scale (the typical wavelength of a thermal radiation quantum) becomes arbitrarily large compared to the Planck length $(\hbar G)^{1 / 2}$. 


\subsection{The Electric Charge Projection}

We will now describe how the Euclidean path integral method is used to evaluate the partition function in a particular charge sector of an abelian gauge theory. At first, we will consider quantum field theory on flat spacetime (no gravity). The extension to include gravity will be discussed later.

Let us briefly recall the standard method of deriving the path integral expression for the partition function of a gauge theory. ${ }^{[31]}$ We work in the the gauge $A_{0}=0$ and evaluate

$$
Z(\beta)=\operatorname{tr}\left(e^{-\beta H}\right)
$$

by summing over the basis of eigenstates of $A_{i}(\vec{x})$ and $\phi(\vec{x})$ (where $\phi$ denotes the matter fields). However, since only physical states are to be included in the sum, we must also insert a projection onto states that satisfy the Gauss law constraint. We thus obtain

$$
Z(\beta)=\int d A_{i}(\vec{x}) d \phi(\vec{x}) d \Omega(\vec{x})\left\langle A^{\Omega}, \phi^{\Omega}\left|e^{-\beta H}\right| A, \phi\right\rangle
$$

where $\Omega(\vec{x})$ is a (time-independent) local gauge transformation. It is important to notice that, since the Gauss law constraint only requires that physical states are invariant under gauge transformations of compact support, $\Omega(\vec{x})$ is restricted in eq. (3.3) to obey

$$
\Omega(\vec{x}) \rightarrow 1 \text { as }|\vec{x}| \equiv r \rightarrow \infty
$$

Now up to a factor of the volume of the local gauge group (which must be removed by gauge fixing), eq. (3.3) may be reexpressed as

$$
Z(\beta)=\int_{\beta \hbar} d A_{\mu} d \phi \exp \left(-S_{E}[A, \phi] / \hbar\right)
$$

where the histories $A_{\mu}(\tau, \vec{x}), \phi(\tau, \vec{x})$ are required to be periodic in Euclidean time 
$\tau$ with period $\beta \hbar$. Furthermore, as a consequence of eq. (3.4), $A_{\tau}$ must satisfy

$$
A_{\tau}(\tau, \vec{x}) \rightarrow 0 \text { as } r \rightarrow \infty .
$$

(We can recover eq. (3.3) and eq. (3.4) from eq. (3.5) and eq. (3.6) by imposing the temporal gauge condition $A_{\tau}=0$.)

The partition function $Z$ defined by eq. (3.5) includes a sum over all physical states. But if the Hilbert space of the theory contains superselection sectors, we may wish to consider $Z$ restricted to a single sector. This requires that further projection operators be inserted in the path integral.

Let us specialize now to the case of an abelian gauge theory (in the Coulomb phase), and construct the partition function restricted to the states of specified electric charge $Q$. A state in the charge- $Q$ sector satisfies

$$
U(\Omega)|Q\rangle=e^{i \omega Q / \hbar e}|Q\rangle,
$$

where the gauge transformation $\Omega(\vec{x})$ has the asymptotic form

$$
\Omega(\vec{x}) \rightarrow e^{i \omega}=\text { constant }, \quad \text { as } r \rightarrow \infty,
$$

and $U(\Omega)$ is the unitary operator that represents this gauge transformation. The projection operator onto physical states of charge $Q$ is

$$
P_{Q}=\int_{0}^{2 \pi} \frac{d \omega}{2 \pi} \int d \Lambda(\vec{x}) e^{-i \omega Q / \hbar e} U\left(e^{i(\Lambda+\omega)}\right)
$$

where

$$
e^{i \Lambda(\vec{x})} \rightarrow \mathbf{1} \text { as } r \rightarrow \infty .
$$


Thus, the partition function in the charge- $Q$ sector may be expressed as

$$
\begin{aligned}
Z(\beta, Q) & =\operatorname{tr}\left(P_{Q} e^{-\beta H}\right) \\
& =\int_{0}^{2 \pi} \frac{d \omega}{2 \pi} e^{-i \omega Q / \hbar e} \int d \Lambda(\vec{x})\left\langle A-\frac{1}{e} \partial \Lambda, e^{i(\Lambda+\omega)} \phi\left|e^{-\beta H}\right| A, \phi\right\rangle \\
& =\int_{0}^{2 \pi} \frac{d \omega}{2 \pi} e^{-i \omega Q / \hbar e} \hat{Z}(\beta, \omega)
\end{aligned}
$$

Here,

$$
\hat{Z}(\beta, \omega)=\int_{\beta \hbar, \omega} d A_{\mu} d \phi \exp \left(-S_{E}[A, \phi] / \hbar\right)
$$

is (up to gauge fixing) the Euclidean path integral over configurations that are periodic in $\tau$ with period $\beta \hbar$, and also satisfy the constraint

$$
\exp \left(i e \int_{0}^{\beta \hbar} d \tau A_{\tau}(\tau, \vec{x})\right)=e^{i \omega}, \text { for } r=\infty
$$

Note that eq. (3.13) constrains only the noninteger part of $(e / 2 \pi) \int d \tau A_{\tau}$. By combining the sum over the integer part with the $\omega$ integral, we may extend the range of integration to $(-\infty, \infty)$, and rewrite eq. (3.11) as

$$
Z(\beta, Q)=\int_{-\infty}^{\infty} \frac{d \omega}{2 \pi} e^{-i \omega Q / \hbar e} Z(\beta, \omega)
$$

where now $Z(\beta, \omega)$ is defined so that the gauge field is restricted by

$$
\left.e \int_{0}^{\beta \hbar} d \tau A_{\tau}(\tau, \vec{x})\right|_{r=\infty}=\omega
$$

This is our result for the partition function in a sector of specified charge. 


\subsection{The Schwarzschild Black Hole}

Let us now briefly review the Euclidean path integral analysis of the thermodynamics of an uncharged black hole in the semiclassical limit, as originally performed by Gibbons and Hawking. ${ }^{[32]}$

If thermal fluctuations in the geometry are to be included, eq. (3.5) can be extended to include an integral over the (Euclidean) spacetime metric. The integral over metrics divides into topologically distinct sectors. The "trivial" sector, which includes small fluctuations about flat space, is probed by integrating over Euclidean geometries that have the topology $R^{3} \times S^{1}$. Gibbons and Hawking argued that the thermodynamics of a black hole with temperature $\beta^{-1}$ can be studied by summing over geometries that have topology $R^{2} \times S^{2}$, are asymptotically flat, and are periodic in imaginary time $\tau$ with period $\beta \hbar$.

To check this hypothesis, we should verify that the standard results of black hole thermodynamics can be recovered from this prescription in the semiclassical limit. In this limit, the integral

$$
Z(\beta) \equiv e^{-\beta F}=\int_{\beta \hbar} e^{-S_{E} / \hbar}
$$

in a given topological sector is dominated by the solution to the Euclidean field equations in that sector that has the lowest Euclidean action. Our task, then, is to find that solution and to evaluate its action.

The crucial observation, now, is that the Euclidean section of the Schwarzschild geometry has the $R^{2} \times S^{2}$ topology, and is periodic in imaginary time. To see this, consider the somewhat more general case of a static spherically symmetric Euclidean geometry, with metric

$$
d s^{2}=e^{2 \Phi} d \tau^{2}+e^{2 \Lambda} d r^{2}+r^{2} d \Omega^{2}
$$

where $\Phi$ and $\Lambda$ are functions of $r$ only. Suppose that $e^{2 \Phi}>0$ and $e^{2 \Lambda}>0$ for $r>r_{+}$, and $e^{2 \Phi}=0$ for $r=r_{+}$. Thus, $r=r_{+}$is the location of an event horizon in 
the Lorentzian continuation of this geometry. In the vicinity of $r=r_{+}$this metric can be rewritten in the form

$$
d s^{2}=\left(\left.\left(e^{\Phi}\right)^{\prime} e^{-\Lambda}\right|_{r=r_{+}}\right)^{2} R^{2} d \tau^{2}+d R^{2}+r^{2} d \Omega^{2}
$$

where $R=0$ at $r=r_{+}$, and the prime denotes differentiation with respect to $r$. We see that $\tau$ can be interpreted as the angular coordinate on the $R-\tau$ plane. But a singularity at $R=0$ can be avoided only if $\tau$ is a periodic variable with period $\beta \hbar$, where

$$
2 \pi(\beta \hbar)^{-1}=\left.\left(e^{\Phi}\right)^{\prime} e^{-\Lambda}\right|_{r=r_{+}}
$$

If this condition is satisfied, then the Euclidean metric eq. (3.17), with $r \geq r_{+}$is topologically $R^{2} \times S^{2}$; it is the analytic continuation of that part of the Lorentzian geometry that lies outside or at the event horizon, and the two-sphere at the origin $R=0$ is the horizon two-sphere. By computing the proper acceleration of static observers near the event horizon of the Lorentzian geometry, and using eq. (3.19), we find

$$
\kappa \equiv a_{\text {proper }} e^{\Phi}=2 \pi / \beta \hbar
$$

which is the relation between the surface gravity $\kappa$ and the black hole temperature $\beta^{-1}$ discovered by Hawking. ${ }^{[2]}$

In the special case of the Euclidean Schwarzschild solution we have

$$
e^{2 \Phi}=e^{-2 \Lambda}=1-\frac{2 G M}{r}
$$

thus, $r_{+}=2 G M, \kappa=1 / 4 G M$, and $\beta \hbar=8 \pi G M$.

Now we turn to the evaluation of the action of this solution. This calculation is somewhat delicate, for two reasons. First, the Euclidean Einstein-Hilbert action includes a boundary term, and the action of the Schwarzschild solution arises solely from this boundary contribution. Second, the boundary term is formally infinite; to define it, we must perform an appropriate subtraction. 
The gravitational action has the form

$$
S_{\text {grav }}=-\frac{1}{16 \pi G} \int d^{4} x \sqrt{g} R+S_{\text {boundary }}
$$

The first term evidently vanishes for a solution to the vacuum Einstein equations, or for a solution such that the stress tensor of the matter has a vanishing trace. In such cases only the boundary term contributes.

For later reference, we will describe the evaluation of the boundary term for the general static, spherically symmetric geometry, eq. (3.17). We will suppose that the geometry is asymptotically flat, and will take the boundary to be $r=\infty$. But since a subtraction will be required, we will first suppose that the boundary is at a finite radius $r$, and will take the $r \rightarrow \infty$ limit only after subtracting.

In this case, the boundary term can be written as

$$
S_{\text {boundary }}=-\frac{1}{8 \pi G} \partial_{\text {normal }} \text { (volume of boundary) ; }
$$

this is the rate, per unit of proper distance, at which the volume of the boundary increases as the boundary is displaced in a direction along the outward-pointing vector normal to the boundary. The "boundary" at radius $r$ is an $S^{1} \times S^{2}$ with

$$
\text { volume }=\left(e^{\Phi(r)} \beta \hbar\right)\left(4 \pi r^{2}\right)
$$

and so we find

$$
S_{\text {boundary }}=\left(-\frac{\beta \hbar}{2 G}\right) e^{-\Lambda(r)}\left(r^{2} e^{\Phi(r)}\right)^{\prime}
$$

This expression diverges linearly as $r \rightarrow \infty$.

The prescription given by Gibbons and Hawking is to subtract the action of a flat spacetime with the same induced geometry at the boundary. In the present case, the flat spacetime has the topology $R^{3} \times S^{1}$, and the proper circumference of the circle is $e^{\Phi(r)} \beta \hbar$. Thus, the "temperature" of the flat spacetime matches the red shifted temperature that would be measured by a static observer at radius $r$ in the curved spacetime. 
The matching flat geometry has action

$$
S_{\text {boundary }}^{(f l a t)}=\left(-\frac{\beta \hbar}{2 G}\right) e^{\Phi(r)}\left(r^{2}\right)^{\prime} .
$$

If the functions $e^{2 \phi}$ and $e^{2 \Lambda}$ appearing in the metric have the asymptotic large- $r$ expansions

$$
e^{2 \Lambda} \sim 1+\frac{2 A}{r}, \quad e^{-2 \Phi} \sim 1+\frac{2 B}{r},
$$

then the subtracted action is

$$
S_{\text {boundary }}-S_{\text {boundary }}^{(f l a t)}=-\frac{\beta \hbar}{2 G}(B-2 A) .
$$

Finally, if eq. (3.17) asymptotically solves the vacuum Einstein equations, then $A=B=G M$, where $M$ is the ADM mass, and we have

$$
S_{\text {boundary }}-S_{\text {boundary }}^{(\text {flat })}=\frac{1}{2} \beta \hbar M .
$$

Now we perform the semiclassical evaluation of the free energy of a Schwarzschild black hole by saturating the path integral with the Euclidean Schwarzschild solution. We have

$$
e^{-\beta F} \simeq e^{-S_{\text {Schwarzschild } / \hbar}},
$$

or

$$
\beta F(\beta)=\frac{1}{2} \beta M=4 \pi \frac{(G M)^{2}}{\hbar G}=\frac{\hbar}{16 \pi G} \beta^{2},
$$

where we have used the relation $\beta \hbar=8 \pi G M$ that was derived from eq. (3.19). From this equation, we may also obtain the entropy

$$
\text { entropy }=\beta M-\beta F(\beta)=4 \pi(G M)^{2} / \hbar G \text {, }
$$

which agrees with the Bekenstein-Hawking value. 


\subsection{The Electrically Charged Black Hole}

We now proceed to the semiclassical evaluation of the thermodynamic functions for an electrically charged black hole.

The action is now

$$
S=S_{\text {grav }}+S_{\text {em }}
$$

where

$$
S_{\mathrm{em}}=\frac{1}{16 \pi} \int d^{4} x \sqrt{g} F_{\mu \nu} F_{\lambda \sigma} g^{\mu \lambda} g^{\nu \sigma}
$$

(We ignore, for now, the charged matter fields.) The stationary point of the action in the sector with $R^{2} \times S^{2}$ topology that is periodic in imaginary time with period $\beta \hbar$ and obeys the constraint eq. (3.15) is the Euclidean Reissner-Nordström solution, namely

$$
\begin{gathered}
A_{\tau}=\frac{\omega}{\beta \hbar e}\left(1-\frac{r_{+}}{r}\right), \quad F_{r \tau}=\frac{\omega}{\beta \hbar e} \frac{r_{+}}{r^{2}}, \\
e^{\Phi+\Lambda}=1, \\
e^{\Phi-\Lambda}=1-\left[1-G\left(\frac{\omega}{\beta \hbar e}\right)^{2}\right]\left(\frac{r_{+}}{r}\right)-G\left(\frac{\omega}{\beta \hbar e}\right)^{2}\left(\frac{r_{+}}{r}\right)^{2} .
\end{gathered}
$$

From eq. (3.19) we determine $r_{+}$to be

$$
r_{+}=\frac{\beta \hbar}{4 \pi}\left[1+G\left(\frac{\omega}{\beta \hbar e}\right)^{2}\right]
$$

Since the electromagnetic stress-tensor is traceless, the gravitational contribution to the action arises solely from the boundary term; evaluating it using eq. (3.28), 
we find

$$
\begin{aligned}
S_{\text {grav }}-S_{\text {grav }}^{(\text {flat })} & =\frac{\beta \hbar}{4 G}\left[1-G\left(\frac{\omega}{\beta \hbar e}\right)^{2}\right] r_{+} \\
& =\frac{(\beta \hbar)^{2}}{16 \pi G}\left[1-G^{2}\left(\frac{\omega}{\beta \hbar e}\right)^{4}\right] .
\end{aligned}
$$

The electromagnetic contribution to the action is

$$
\begin{aligned}
S_{\mathrm{em}} & =\frac{1}{2} \beta \hbar\left(\frac{\omega}{\beta \hbar e}\right)^{2} r_{+} \\
& =\frac{(\beta \hbar)^{2}}{8 \pi}\left(\frac{\omega}{\beta \hbar e}\right)^{2}\left[1+G\left(\frac{\omega}{\beta \hbar e}\right)^{2}\right] .
\end{aligned}
$$

Combining the two contributions gives

$$
S-S^{(f l a t)}=\frac{(\beta \hbar)^{2}}{16 \pi G}\left[1+G\left(\frac{\omega}{\beta \hbar e}\right)^{2}\right]^{2} .
$$

Now we project out the contribution from the charge $Q$ sector by integrating over $\omega$ as in eq. (3.14), obtaining

$$
\begin{aligned}
& \exp [-\beta F(\beta, Q)]=\int_{-\infty}^{\infty} \frac{d \omega}{2 \pi} \exp \left[-i \frac{\omega Q}{\hbar e}\right] \\
& \times \exp \left\{-\frac{1}{\hbar} \frac{(\beta \hbar)^{2}}{16 \pi G}\left[1+G\left(\frac{\omega}{\beta \hbar e}\right)^{2}\right]^{2}\right\} .
\end{aligned}
$$

In the semiclassical limit, $\hbar$ approaches zero with $\beta \hbar, Q, G$, and $e$ held fixed. Thus, the $\omega$ integral can be evaluated in the steepest-descent approximation. The $\omega$-contour is deformed so that it passes through a saddle point on the imaginary 
axis. (The contour is not rotated; its ends must be fixed, or else $Z(\beta, \omega)$ will blow up.) The semiclassical expression for the free energy becomes

$$
F(\beta, Q)=\left.(\Omega(\beta, \Phi)+Q \Phi)\right|_{\text {stationary }},
$$

where we have defined a new dummy variable

$$
\Phi=\frac{i \omega}{\beta \hbar e},
$$

and

$$
\Omega(\beta, \Phi)=\frac{\beta \hbar}{16 \pi G}\left(1-G \Phi^{2}\right)^{2} .
$$

We see that eq. (3.42) may be interpreted as a Legendre transform; $\Phi$ is the "chemical potential" coupled to the electric charge $Q$, and $\Omega(\beta, \Phi)$ is the associated thermodynamic potential.

The value of $\Phi$ at the saddle point is $\Phi=Q / r_{+}$; this can be interpreted as the electrostatic potential difference between the black hole horizon and spatial infinity, for a black hole with electric charge $Q$ and radius $r_{+}$. In fact, the solution given by eq. (3.35)-(3.37), with $\Phi$ assuming this saddle-point value, is the analytic continuation to imaginary time of the Reissner-Nordström black hole solution with electric charge $Q$. This continued solution has imaginary $F_{r \tau}$, and negative electromagnetic action.

An alternative way to describe the semiclassical calculation, then, is as follows: If the electrically charged Reissner-Nordstrom black hole solution is continued to imaginary time, the Euclidean action of the continued solution, divided by $\beta \hbar$, is the thermodynamic potential $\Omega(\beta, \Phi)$ of the black hole. This is the result found by Gibbons and Hawking. ${ }^{[32]}$

The more familiar formulas of black hole thermodynamics can be recovered from eq. (3.44) by Legendre transformations and changes of variable. For example, 
we may write

$$
\beta \Omega=\frac{1}{\hbar}\left(S-S^{(\text {flat })}\right)=\frac{1}{2} \beta(M-Q \Phi)
$$

where the first term is the gravitational action and the second term is the electromagnetic action. ( $M$ is the black hole mass.) Therefore,

$$
\beta F=\frac{1}{2} \beta(M+Q \Phi)=\frac{1}{\hbar}\left(S_{\text {grav }}-S_{\text {grav }}^{(\text {flat })}\right)-\frac{1}{\hbar} S_{\mathrm{em}},
$$

and

$$
\text { entropy }=\beta M-\beta F=\frac{1}{\hbar}\left(S-S^{(\text {flat })}\right) \text {. }
$$

From

$$
M=\frac{\beta \hbar}{8 \pi G}\left(1-G^{2} \Phi^{4}\right)
$$

and

$$
Q=\frac{\beta \hbar}{4 \pi} \Phi\left(1-G \Phi^{2}\right)
$$

we then obtain

$$
\beta \hbar=2 \pi G M \frac{(1+X)^{2}}{X}
$$

and

$$
\text { entropy }=\pi \frac{(G M)^{2}}{\hbar G}(1+X)^{2} \text {, }
$$

where we have defined

$$
X \equiv\left(1-\frac{Q^{2}}{G M^{2}}\right)^{1 / 2}=\frac{1-G \Phi^{2}}{1+G \Phi^{2}}
$$




\subsection{The Extreme Solutions}

According to eq. (3.50), the "extreme" Reissner-Nordström black hole with $Q^{2}=G M^{2}$ has vanishing temperature. This result has a simple heuristic interpretation. The electrostatic energy $Q^{2} / 2 r_{+}$stored in the electric field (outside the event horizon) becomes comparable to the mass $M$ as the extreme limit is approached. Hence there is no mass left over at the center to support the horizon; the surface gravity approaches zero, and with it the Hawking temperature.

In fact, as the charge to mass ratio approaches the extreme value, the inner Cauchy horizon at $r=r_{-}<r_{+}$approaches the event horizon at $r=r_{+}$. The black hole is dangerously close to becoming a naked singularity. In this context, the cosmic censorship hypothesis coincides with the third law of thermodynamics. The extreme black hole appears to be a stable object, unable to shed mass by the Hawking process.

So far, though, we have neglected the coupling of the electromagnetic field to electrically charged particles. (A coupling to particles with charge $\hbar e$ was implicit, in the construction of the charge projection operator.) The charged particles have no effect on the leading semiclassical analysis described above, but have important effects that are higher order in $\hbar$.

The electrostatic potential at the horizon of an extreme black hole is

$$
\Phi=G^{-1},
$$

(if $Q$ is positive) and so the electrostatic potential energy of an elementary particle with charge $q$ at the horizon is $q \Phi=q / G$. In the real world, then, a positron at the horizon has enormous electrostatic energy compared to its mass $m$. Hence, dielectric breakdown of the vacuum occurs outside the horizon. It is energetically favorable to produce an $\mathrm{e}^{+} \mathrm{e}^{-}$pair, allowing the electron to be absorbed by the black hole while the positron is ejected to spatial infinity with an ultrarelativistic velocity. By this process, the black hole neutralizes its charge and reduces its mass. $^{[33]}$ 
As a matter of principle, though, we are free to contemplate a fictitious world such that the mass $m$ and charge $q$ of all elementary particles obey

$$
G m^{2}>q^{2}
$$

In this world, the objects with the smallest ratio of mass to charge are extreme black holes. Since electric charge is conserved, the decay of these objects to elementary particles is kinematically forbidden.

The only decay channel that is potentially available to an extreme charged black hole is a state that contains black holes of lower charge and mass. At the classical level, all extreme black holes have precisely the same charge-to-mass ratio, so this channel is just marginally forbidden. We are obligated, then, to consider the quantum corrections that might renormalize this ratio. There are supersymmetric models in which it is known that no renormalization occurs; in these models, the extreme black holes are (just barely) stable. ${ }^{[34]}$ Stable extreme black holes are fairly generic even in non-supersymmetric models. The reason is that the charge appearing in the relation $Q^{2}=G M^{2}$ is really the charge of the black hole renormalized at a length scale comparable to $r_{+}$, while the charge that enters the condition for kinematic stability is the charge renormalized at a large length scale. If $r_{+}$is small compared to the Compton wavelength $\hbar / m$ (and this is possible for a black hole that is much heavier than the Planck mass as long as $\left.q^{2} / \hbar<<1\right)$, then the charge is more effectively screened by vacuum polarization for a small black hole than for a large one. The lighter extreme black holes, therefore, have more mass per unit charge than the heavier holes, and the heavier holes are unable to decay.

These models that contain absolutely stable extreme black holes are a very intriguing subject for further investigation. According to eq. (3.51), the extreme holes have a large intrinsic entropy. Nonvanishing entropy at zero temperature ordinarily indicates a degenerate ground state, and so we are challenged to understand the nature of the degenerate states. Furthermore, since the extreme black 
holes are stable particles, the issue of loss of quantum coherence comes into sharper focus. One might hope to construct an $S$-matrix that describes the scattering of elementary particles off of an extreme black hole. But if such scattering processes inevitably destroy quantum-mechanical phase information, then no such $S$-matrix should exist.

\subsection{Magnetically Charged Black Hole}

The semiclassical analysis of the thermodynamics of a magnetically charged black hole is easier than the analysis of an electrically charged black hole, because the magnetic charge projection is simpler than the electric charge projection.

To project out states with magnetic charge $P$, we merely restrict the path integral to configurations that have magnetic flux $4 \pi P$ on the two-sphere at $r=\infty$. The semiclassical evaluation of the partition function in this sector is dominated by the magnetically charged Euclidean Reissner-Nordström solution, which is the analytic continuation to imaginary time of the Lorentzian magnetically charged solution. This Euclidean solution has the same geometry (and gravitational action) as the continuation of the electrically charged Reissner-Nordström solution, but it has real $F_{\theta \phi}$ and positive electromagnetic action. In the semiclassical limit, we find

$$
\beta F(\beta, P)=\frac{1}{\hbar}\left(S_{\text {grav }}-S_{\text {grav }}^{(\text {flat })}\right)+\frac{1}{\hbar} S_{\mathrm{em}} .
$$

Since the analytically continued electrically charged and magnetically charged solutions have electromagnetic action of opposite sign, eq. (3.55) is the same function of $\beta$ and $P$ as the function of $\beta$ and $Q$ in eq (3.46). Thus, the magnetically charged and electrically charged black holes have identical thermodynamics. Of course, this agrees with the standard analysis; ${ }^{[7]}$ the black holes with electric charge $Q$ and magnetic charge $P=Q$ have the same surface gravity and surface area.

We find, then, that the Euclidean action of the magnetic Euclidean ReissnerNordström solution is $\beta \hbar F(\beta, P)$, where $F$ is the free energy, while the Euclidean 
action of the electric Euclidean Reissner-Nordström solution is $\beta \hbar \Omega(\beta, \Phi)$, where $\Omega$ is the thermodynamic potential. This agrees with the analysis of Gibbons and Hawking.

\subsection{Magnetically Charged Black Holes in Yang-Mills Theory}

The magnetically charged Reissner-Nordström black hole is also a solution to the field equations of Yang-Mills theory coupled to gravity.

Consider, for example, the 't Hooft-Polyakov model ${ }^{[28]}$ in which $S O(3)$ is spontaneously broken to $U(1)$ by the Higgs mechanism. This model, coupled to gravity, actually contains three types of magnetic monopole solutions, at least if the symmetry-breaking mass scale is well below the Planck mass.

The first (light) type is essentially the 't Hooft-Polyakov solution, slightly perturbed by gravitational effects. It is non-singular and has no event horizon; the $S O(3)$ gauge symmetry is restored in its core. The second (heavy) type is the magnetically charged Reissner-Nordström solution black hole. In this solution, there is no sign of symmetry restoration near the event horizon. The Higgs field is covariantly constant everywhere outside the horizon. ${ }^{[35]}$

The third type of solution was discovered only very recently. ${ }^{[36]}$ It may be described as a small black hole embedded inside the core of a magnetic monopole. For a sufficiently small black hole, the Reissner-Nordström solution is classically unstable. The solution of the third type, which has a nontrivial Higgs field core outside the horizon, is favored instead. The condition for classical stability of the Reissner-Nordström solution is $r_{+} \geq \sqrt{n} \mu_{V}^{-1}$, where $n$ is the magnetic charge in units of $1 / e$, and $\mu_{V}^{-1}$ is the Compton wavelength of the vector meson that acquires mass via the Higgs mechanism. Extreme solutions of the third type do not existblack holes inside monopoles always have a finite temperature and emit Hawking radiation. The extreme Reissner-Nordström solution is classically stable only if $n \geq e^{2} /\left(G \mu_{V}^{2}\right)$. 
We may also consider a model in which the unbroken gauge symmetry is a nonabelian group $\mathrm{H}$. There will again be both nonsingular monopoles and (two types of) magnetically charged black holes. Suppose, to be definite, that the unbroken gauge group is $H=S U(N) / Z_{N}$; that is, all fields transform trivially under the center $Z_{N}$ of $S U(N)$. The magnetic field of an $S U(N)$ magnetic monopole can be written in the form

$$
\mathbf{F}_{\theta \phi}=\frac{1}{2 e} \hat{\mathbf{P}} \sin \theta
$$

where $e$ is the gauge coupling; $\hat{\mathbf{P}}$ is a matrix in the Lie algebra of $S U(N)$ that, in order to satisfy the Dirac quantization condition, must be such that

$$
\exp (2 \pi i \hat{\mathbf{P}})=e^{2 \pi i n / N} \mathbf{1} \in Z_{N}
$$

This element of the center is the topological magnetic charge of the monopole. ${ }^{[29]}$

For each nonzero value of the $Z_{N}$ charge $n=1,2,3, \ldots, N-1$, there is a unique magnetic field configuration that is classically stable.$^{[29,37]}$ In this configuration (and in a particular gauge), the matrix $\hat{\mathbf{P}}$ is the diagonal matrix

$$
\hat{\mathbf{P}}=\hat{\mathbf{P}}_{\mathbf{n}} \equiv \operatorname{diag}(\underbrace{\frac{n}{N}, \ldots, \frac{n}{N}}_{N-n \text { times }}, \underbrace{\frac{n-N}{N}, \ldots, \frac{n-N}{N}}_{n \text { times }})
$$

Of course, if $S U(N)$ is unbroken, then we expect this theory to be confining, and the classical magnetic field will be screened at long range. We will further consider the consequences of this screening in Section 5. For now, we merely note that, since the $Z_{N}$ magnetic charge has a topological meaning, it is easy to construct a projection onto a sector with a definite value of the $Z_{N}$ charge, by restricting the path integral to field configurations with a specified $Z_{N}$ flux on the two-sphere at $r=\infty$. The black hole partition function in each nontrivial sector, in the semiclassical limit, is dominated by the stable classical black hole 
solution - either the Euclidean Reissner-Nordström solution, with the magnetic field given by eq. (3.56) and eq. (3.58), or, if the Reissner-Nordström solution is unstable, a solution analogous to that described in Ref. 36. (But effects that are nonperturbative in $\hbar$ can drastically change the story, as we will see in Section 5.)

To complete this classical discussion, we will find the mass of the extreme Reissner-Nordström black hole with $Z_{N}$ charge $n$. The easiest way to do this is to apply the result that the extreme black holes in the abelian theory have $G M^{2}=P^{2}$; acting as a source in the Einstein equations, the field of an $S U(N)$ monopole with charge $n$ is equivalent to that of a $U(1)$ monopole with a charge $P_{n}$ that we can compute. ${ }^{[38]}$ The conventional normalization of the $S U(N)$ generators (and of the gauge coupling $e)^{\star}$ is such that $\operatorname{tr} \mathbf{F}_{\theta \phi}^{2}$ in the nonabelian theory is equivalent to $\frac{1}{2} F_{\theta \phi}^{2}$ in the abelian theory. Hence, the mass $M_{n}$ of the extreme black hole with $Z_{N}$ charge $n$ is given by

$$
G M_{n}^{2}=P_{n}^{2} \equiv \frac{1}{2 e^{2}} \operatorname{tr} \hat{\mathbf{P}}^{2}=\left(\frac{1}{2 e^{2}}\right) \frac{n(N-n)}{N}, \quad n=1,2, \ldots, N-1
$$

If the dimensionless coupling constant $\hbar e^{2}$ (renormalized at a distance scale comparable to the size of the black hole) is small, then $M_{n}$ is much larger than the Planck mass, so that semiclassical methods are reliable.

If there are light nonsingular monopoles that carry the $Z_{N}$ charge, then the extreme Reissner-Nordström solution will be classically unstable. ${ }^{[36]}$ But if the symmetry breaking mass scale is large enough, then the $(n= \pm 1)$ magnetically charged Reissner-Nordström black hole is the lightest magnetically charged object in the theory, and must be absolutely stable. In fact, in that event, the magnetically charged black hole is stable for each value of the charge $n$, for according to eq. (3.59), there is no lighter object with the same total magnetic charge.

\footnotetext{
* But see the remark about the normalization of $e$ in Section 4.2 below.
} 


\section{Screened Electric Charge}

\section{1. $Z_{N}$ Electric Charge Projection}

We will now consider an abelian Higgs model in which $U(1)$ is spontaneously broken to $Z_{N}$, as described in Section 2. $Z_{N}$ electric charge is screened, and so, according to the no-hair theorem, the $Z_{N}$ electric charge on a stationary black hole has no effect on the geometry of the hole, in the classical approximation. But the charge does have quantum effects, effects that, as we will see, are nonperturbative in $\hbar$. We wish to calculate how the $Z_{N}$ electric charge modifies the thermodynamic behavior of the black hole. ${ }^{[25,26]}$

In this model, the charge superselection sectors are labeled by the $Z_{N}$ charge, and our first task is to find the Euclidean path integral prescription for computing the partition function restricted to a particular charge sector. For this purpose, we note that the periodic configurations of finite action must be such that

$$
\left.e \int_{0}^{\beta \hbar} d \tau A_{\tau}(\tau, \vec{x})\right|_{r=\infty}=\frac{2 \pi}{N} k,
$$

where $k$ is an integer. (Otherwise, the contribution to the action arising from the covariant derivative of the Higgs field is divergent.) Thus, the integral over $\omega$ in eq. (3.14) collapses to a sum over $k$. We therefore find that

$$
Z(\beta, Q)=\frac{1}{N} \sum_{k=-\infty}^{\infty} e^{-2 \pi i k Q / N \hbar e} Z(\beta, k)
$$

where

$$
Z(\beta, k)=\int_{\beta \hbar, k} e^{-S_{E} / \hbar}
$$

is the Euclidean path integral over configurations that are periodic in Euclidean time with period $\beta \hbar$ and satisfy the constraint eq. (4.1). Evidently, $Z(\beta, Q)$ depends only on $Q$ modulo $N \hbar e$, the charge that is not screened by the condensate. 
An alternative way to derive eq. (4.2) is to repeat the derivation in Section 3.3, but inserting the $Z_{N}$ charge-projection operator

$$
P_{Q}=\frac{1}{N} \sum_{k=0}^{N-1} e^{-2 \pi i k Q / N \hbar e} U(k)
$$

into the path integral, where $U(k)$, the operator that represents a local $Z_{N}$ transformation, acts on a charge- $Q$ state according to

$$
U(k)|Q\rangle=e^{2 \pi i k Q / N \hbar e}|Q\rangle
$$

If we are interested in the case of a black hole with specified $Z_{N}$ electric charge, then we integrate over the geometry as well, restricted to the sector with topology $R^{2} \times S^{2}$. In this context, eq. (4.1) has a rather remarkable interpretation. If we regard $F_{r \tau}$ as a magnetic field, then $\int_{0}^{\beta \hbar} d \tau A_{\tau}=\int d r d \tau F_{r \tau}$ is the magnetic flux in the $r-\tau$ plane, and $k$ is the vorticity, the value of the flux in units of the flux quantum $2 \pi / N e$. The electric charge $Q$ determines the phases that weight the different vorticity sectors. In fact, as we will explain in more detail below, the integer $k$ can be interpreted as the net number of virtual cosmic string world sheets that wrap around the black hole. The phase that accompanies the contribution from the sector with vorticity $k$ in eq. (4.2), then, is precisely the Aharonov-Bohm phase acquired by the virtual string, if the black hole carries charge $Q$.

We will briefly explain how the results of this section can be generalized to the case in which the unbroken local symmetry group is a nonabelian finite group $H$. In this case, an operator can be constructed that projects out states that transform as a specified irreducible representation $(\mu)$ of $H$; this projection operator is

$$
P_{(\mu)}=\frac{n_{\mu}}{n_{H}} \sum_{h \in H} \chi^{(\mu)}(h)^{*} U(h)
$$

Here $U(h)$ is the operator that represents $h \in H$ acting on Hilbert space, $n_{H}$ is the order of the group, $\chi^{(\mu)}$ is the character of the irreducible representation $(\mu)$, 
and $n_{\mu}$ is the dimension of $(\mu)$. By inserting this projection operator into the path integral, we obtain an expression for the partition function restricted to the charge sector containing states that transform according to $(\mu)$. The result is

$$
Z(\beta,(\mu))=\frac{n_{\mu}^{2}}{n_{H}} \sum_{h \in H} \frac{1}{n_{\mu}} \chi^{(\mu)}(h)^{*} Z(\beta, h)
$$

where $Z(\beta, h)$ is the Euclidean path integral over configurations that are periodic in Euclidean time with period $\beta \hbar$ and satisfy the constraint

$$
P \exp \left(\left.i g \int_{0}^{\beta \hbar} d \tau A_{\tau}(\tau, \vec{x})\right|_{r=\infty}\right)=h .
$$

For the case of a black hole with $H$-charge, the constraint requires a vortex with "flux" $h \in H$ to occupy the $r$ - $\tau$ plane. Once again, the interpretation is clear. The factor $\left(1 / n_{\mu}\right) \chi^{(\mu)}(h)^{*}$ that weights the sector with vortex flux $h$ is precisely the Aharonov-Bohm factor acquired by a virtual cosmic string with flux $h$ that winds around the black hole. The reason that this "phase" has modulus less than one is that a string that is initially uncharged may exchange charge with the black hole during the winding, and the virtual string can re-annihilate only if it remains uncharged. The probability that the string remains uncharged after winding around an object that carries charge $(\mu)$ is just $\left|\left(1 / n_{\mu}\right) \chi^{(\mu)}(h)\right|^{2}$, the absolute value squared of the weight factor appearing in eq. $(4.7){ }^{[39]}$

\subsection{Semiclassical Thermodynamics}

As in Section 3, we find the semiclassical thermodynamic behavior of a black hole with $Z_{N}$ electric charge by evaluating eq. (4.2) in the limit $\hbar \rightarrow 0$, with $\beta \hbar$ held fixed. But now, in taking the limit, we will treat the charge $Q$ differently than before. 
In the standard semiclassical limit (invoked in Section 3), the classical charge $Q$ is held fixed as $\hbar$ gets small. In this limit, the charge quantum $\hbar e$ becomes arbitrarily small compared to $Q$. And the Aharonov-Bohm phase $\exp (-2 \pi i Q / N \hbar e)$, acquired when charge $Q$ circumnavigates flux $2 \pi / N e$, oscillates wildly. The oscillations wipe out the Aharonov-Bohm interference; the Aharonov-Bohm effect (which is quantum mechanical) does not survive in the standard classical limit.

We are interested in a different case, in which the $Z_{N}$ charge is held fixed as $\hbar$ gets small. Thus, we fix $Q / N \hbar e$, the classical charge in units of the charge of the condensate, rather than $Q$. Then the Aharonov-Bohm phases appearing in eq. (4.2) are well-behaved, and effects that depend on the $Z_{N}$ charge can be investigated.

The model that we are studying has the action

$$
S=S_{\text {grav }}+S_{\mathrm{em}}+S_{\mathrm{Higgs}},
$$

with $S_{\text {grav }}$ as in eq. (3.22), $S_{\text {em }}$ as in eq. (3.34), and ${ }^{\star}$

$$
\begin{aligned}
S_{\mathrm{Higgs}}=\frac{1}{4 \pi} \int d^{4} x \sqrt{g}[ & g^{\mu \nu}\left(\partial_{\mu}-i e N A_{\mu}\right) \phi^{*}\left(\partial_{\nu}+i e N A_{\nu}\right) \phi \\
+ & \left.\frac{\lambda}{2}\left(|\phi|^{2}-\frac{v^{2}}{2}\right)^{2}+\xi|\phi|^{2} R\right] .
\end{aligned}
$$

(We may ignore the fields that carry nontrivial $Z_{N}$ electric charge, for they have no effect on the leading semiclassical result.) Note that we have adopted (with some reluctance) unrationalized units such that the gauge field kinetic term in the Euclidean Lagrange density is $(1 / 8 \pi)\left(E^{2}+B^{2}\right)$. We do so to be consistent with the notation of Section 3, and with most of the literature on charged black holes. (In the rationalized units usually favored by particle physicists, the gauge field kinetic term is $(1 / 2)\left(E^{2}+B^{2}\right)$, and the condition satisfied by an extreme

\footnotetext{
$\star$ In the subsequent discussion, we set $\xi=0$. The results are qualitatively similar for $\xi \neq 0$.
} 
Reissner-Nordström black hole is $Q^{2}=4 \pi G M^{2}$.) Thus we are normalizing the gauge coupling $e$ in a manner than departs from the common convention; the (more common) rationalized coupling $e_{\text {rat }}$ is related to our $e$ by

$$
e^{2}=\frac{e_{\mathrm{rat}}^{2}}{4 \pi}
$$

We wish to caution the reader that, in our units, the "fine structure constant" is $\alpha=\hbar e^{2}=\hbar e_{\text {rat }}^{2} / 4 \pi$.

To evaluate $Z(\beta, k)$ in the semiclassical limit, we seek the solution to the Euclidean field equations, of lowest action, that has topology $R^{2} \times S^{2}$, is asymptotically flat, is periodic in $\tau$ with period $\beta \hbar$, and has vorticity $k$. Since the magnetic flux quantum is independent of $\hbar$, increasing the vorticity increases the action of this solution by an amount that is independent of $\hbar$. Therefore, to compute the leading charge-dependent corrections to the partition function in the semiclassical limit, we need only retain the sectors with vorticity $k=0$ and $k= \pm 1$.

Hence we find

$$
Z(\beta, Q) \simeq \frac{1}{N}\left[Z(\beta, k=0)+2 \cos \left(\frac{2 \pi Q}{N \hbar e}\right) Z(\beta, k=1)\right]
$$

or

$$
\frac{Z(\beta, Q)}{Z(\beta, Q=0)} \simeq 1-2\left[1-\cos \left(\frac{2 \pi Q}{N \hbar e}\right)\right] \frac{Z(\beta, k=1)}{Z(\beta, k=0)} .
$$

The $k=0$ solution is just the Euclidean Schwarzschild solution, so we have

$$
\frac{Z(\beta, k=1)}{Z(\beta, k=0)} \simeq \frac{1}{2} C(\beta \hbar) e^{-\Delta S_{\text {vortex }} / \hbar}
$$

Here,

$$
\Delta S_{\text {vortex }}=S_{\text {vortex }}-S_{\text {Schwarzschild }}
$$

where $S_{\text {vortex }}$ is the action of the $k=1$ solution, and $(1 / 2) C(\beta \hbar)>0$ is a ratio of functional determinants. (This ratio is positive because the phases that arise from 
the negative modes in the integration over geometries are divided out.) Taking the logarithm of eq. (4.13), we find the leading charge-dependent contribution to the black hole free energy

$$
\beta F(\beta, Q)-\beta F(\beta, Q=0) \simeq\left[1-\cos \left(\frac{2 \pi Q}{N \hbar e}\right)\right] C(\beta \hbar) e^{-\Delta S_{\text {vortex }} / \hbar}
$$

Now we may invoke the thermodynamic identity

$$
M(\beta, Q)=\frac{\partial(\beta F)}{\partial \beta}
$$

to find

$$
M(\beta, Q)-M(\beta, Q=0) \simeq-\left[1-\cos \left(\frac{2 \pi Q}{N \hbar e}\right)\right] \frac{\partial \Delta S_{\text {vortex }}}{\partial(\beta \hbar)} C(\beta \hbar) e^{-\Delta S_{\text {vortex }} / \hbar}
$$

the expression relating the mass, charge, and temperature. (We have neglected a term that is suppressed by an additional power of $\hbar$.)

Note the significance of the sign of $\partial \Delta S_{\text {vortex }} / \partial(\beta \hbar)$. If it is positive, then adding charge reduces the mass of a black hole of given temperature, and correspondingly reduces the temperature of a black hole of given mass. Conversely, if $\partial \Delta S_{\text {vortex }} / \partial(\beta \hbar)$ is negative, then adding charge heats up a black hole of given mass.

To go further, we must calculate $\Delta S_{\text {vortex }}$. Let us assume that the vortex is rotationally invariant, satisfying the Ansatz

$$
\begin{gathered}
\phi=\frac{\rho(r)}{\sqrt{2}} \exp (-2 \pi i \tau / \beta \hbar), \\
e A_{\tau}=\frac{2 \pi}{N \beta \hbar}[1-a(r)],
\end{gathered}
$$


and with the geometry of the form eq. (3.17). Then the action can be expressed as

$$
\begin{aligned}
S_{\text {grav }}-S_{\text {grav }}^{(\text {flat })}= & \beta \hbar \int_{r_{+}}^{\infty} d r\left(-\frac{1}{2 G}\right)\left[e^{(\Phi+\Lambda)}+e^{(\Phi-\Lambda)}\left(2 r \Lambda^{\prime}-1\right)\right] \\
& -\frac{1}{G} \pi r_{+}^{2}+\frac{\beta \hbar}{G} \lim _{r \rightarrow \infty}\left[r e^{\Phi}\left(1-e^{-\Lambda}\right)\right]
\end{aligned}
$$

(where we have done an integration by parts, and used eq. (3.19) and eq. (3.25)$(3.26))$, and

$$
\begin{gathered}
S_{\text {matter }} \equiv S_{\text {em }}+S_{\text {Higgs }} \\
=\beta \hbar \int_{r_{+}}^{\infty} d r r^{2}\left\{\frac{1}{2}\left(\frac{2 \pi}{N e \beta \hbar}\right)^{2}\left[e^{-(\Phi+\Lambda)}\left(a^{\prime}\right)^{2}+e^{(\Lambda-\Phi)}(N e)^{2} \rho^{2} a^{2}\right]\right. \\
\\
\left.\quad+e^{(\Phi-\Lambda)} \frac{1}{2}\left(\rho^{\prime}\right)^{2}+e^{(\Phi+\Lambda)} \frac{\lambda}{8}\left(\rho^{2}-v^{2}\right)^{2}\right\} .
\end{gathered}
$$

(The primes denote derivatives with respect to $r$.) By demanding that the action is stationary, we obtain coupled differential equations satisfied by $\Phi(r), \Lambda(r), \rho(r)$, and $a(r)$.

In the next two sections, we will describe how these equations can be solved analytically, and the action of the solution explicitly computed, in two different limiting cases. First, though, we remark on how eq. (4.13) generalizes to the case in which the unbroken local symmetry group is a (not necessarily abelian) discrete group $H$. In eq. (4.7), we expressed the partition function $Z(\beta,(\mu))$ of a black hole with $H$-charge $(\mu)$ as a weighted sum of contributions from sectors of specified vorticity. The path integral $Z(\beta, h)$ restricted to the sector with vorticity $h$ actually depends only on the conjugacy class to which $h$ belongs, for $h \rightarrow h^{\prime} h h^{\prime-1}$ under a global $H$ transformation. Furthermore, $C P T$-invariance implies $Z(\beta, h)=$ $Z\left(\beta, h^{-1}\right)$; an $h$ string that wraps in one sense around the black hole is equivalent to an $h^{-1}$ string that wraps in the opposite sense. Barring further symmetries or accidental degeneracies, the charge dependence of $Z(\beta,(\mu))$ will be dominated in 
the semiclassical limit by vortices belonging to a single class (and the inverse class), the vortices of lowest Euclidean action. If we denote the class that dominates by $\alpha$, then the generalization of eq. (4.13) becomes

$$
\frac{Z(\beta,(\mu))}{Z(\beta,(\mu)=(0))} \simeq n_{\mu}^{2}\left(1-2 n_{\alpha}\left[1-\frac{1}{n_{\mu}} \operatorname{Re} \chi_{\alpha}^{(\mu)}\right] \frac{Z(\beta, \alpha)}{Z(\beta,\{e\})}\right)
$$

where $n_{\alpha}$ is the order of the class.

\subsection{The Thin String Limit}

We first consider the limit in which the natural thickness $\mu^{-1}$ of the string is much less than the radius $r_{+} \simeq 2 G M$ of the event horizon of the black hole. (Here $\mu^{-1}=(N e v)^{-1}$ is the Compton wavelength of the massive vector meson.) In this limit, the vortex shrinks to a point at the origin of the $r-\tau$ plane. The matter field configuration near the origin looks just like the cross section of a straight cosmic string in flat space. We may interpret this configuration as the world sheet of a virtual string wrapped tightly around the minimal two-sphere (the "horizon") of the Euclidean Schwarzschild geometry.

To start with, we will make an additional assumption, that the string tension is small in Planck units, or

$$
G \tilde{T}_{\text {string }}<<1
$$

Here $\tilde{T}_{\text {string }}$ is the tension of a straight cosmic string in flat space; it has the form

$$
\tilde{T}_{\text {string }}=\frac{1}{4} v^{2} f\left(\lambda / e^{2}\right)
$$

where $f$ is a slowly varying function such that $f(1)=1^{[40]}$ (There is a gravitational correction to eq. (4.25), as the string has a $G$-dependent gravitational self-energy; this correction may be ignored under the assumption eq. (4.24).) 
Now there are two contributions to the action that we must consider - the contribution $S_{\text {matter }}=S_{\mathrm{em}}+S_{\text {Higgs }}$ due to the matter fields, and the gravitational contribution $S_{\text {grav }}$. Since the matter field configuration is a vortex localized on the minimal two-sphere, we have

$$
S_{\text {matter }}=4 \pi r_{+}^{2} \tilde{T}_{\text {string }} .
$$

To estimate $S_{\text {grav }}$, we note that, for $G \tilde{T}_{\text {string }}<<1$, the geometry of the vortex solution is close to the Euclidean Schwarzschild geometry; the perturbation of the

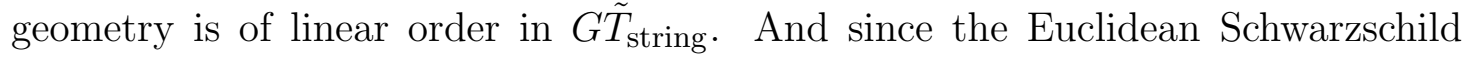
geometry is a stationary point of the Einstein-Hilbert action, the change in the gravitational action is of second order. Thus, we have

$$
\Delta S_{\text {grav }}=\frac{1}{16 \pi G} o\left[\left(G \tilde{T}_{\text {string }}\right)^{2}\right]
$$

which can be neglected compared to $S_{\text {matter }}$. We therefore find

$$
\Delta S_{\text {vortex }} \simeq \frac{(\beta \hbar)^{2}}{4 \pi} \tilde{T}_{\text {string }}, \quad G \tilde{T}_{\text {string }}<<1
$$

In fact, we can go further, and find an expression for $\Delta S_{\text {vortex }}$ in the thin string limit without making the assumption eq. (4.24). We may replace $S_{\text {matter }}$ by an effective action - the Nambu-Goto action for a relativistic string with tension $T_{\text {string }}$; thus the "matter" action becomes

$$
S_{\mathrm{NG}}=\text { (World Sheet Area) } T_{\text {string }} \text {. }
$$

Here $T_{\text {string }}$ is not, in general, the same as $\tilde{T}_{\text {string }}$ given by eq. (4.25), because $T_{\text {string }}$ may include $G$-dependent gravitational corrections. In the vortex solution, eq. (4.29) coincides with eq. (4.26), except that $T_{\text {string }}$ has replaced $\tilde{T}_{\text {string }}$. 
Now consider $S_{\text {grav }}$. The stress tensor of a static straight string along the $z$ axis, integrated over a planar slice perpendicular to the string, has the form

$$
\int d^{2} x \sqrt{h} \theta_{\nu}^{\mu}=T_{\text {string }} \operatorname{diag}(1,0,0,1)
$$

(where $h$ is the induced metric on the slice). From the Einstein equation, we have

$$
R=8 \pi G \theta_{\mu}^{\mu}
$$

and therefore, using eq. (4.30),

$$
-\frac{1}{16 \pi G} \int d^{4} x \sqrt{g} R=-(\text { Area }) T_{\text {string }}
$$

which exactly cancels eq. (4.29).

It remains to compute $S_{\text {boundary }}$. Outside of the pointlike vortex, the geometry is (locally) a Euclidean Schwarzschild solution. But the point vortex can modify the relation between $\beta \hbar$ and $S_{\text {boundary }}$. To find the correct relation, it is helpful to notice that the problem of finding the geometry outside the vortex, but at a distance from the vortex that is much less than $r_{+}$, is equivalent to another problem with a well-known solution - the problem of finding the geometry outside a static straight string. ${ }^{[41]}$ The geometry outside the static straight string is a conical space with a deficit angle

$$
\delta=8 \pi G T_{\text {string }}
$$

It follows that the effect of the point vortex at the origin is to modify the condition eq. (3.19); it is replaced by

$$
2 \pi-\delta=\frac{\beta \hbar}{4 G M},
$$

where $M$ is the mass appearing in the Euclidean Schwarzschild solution that describes the geometry outside the vortex. Now, the boundary contribution to the 
action of the vortex solution is related to $M$ as in eq. (3.29), so we find

$$
S_{\text {vortex }}-S_{\text {grav }}^{(\text {flat })}=\frac{(\beta \hbar)^{2}}{16 \pi G}\left(1-4 G T_{\text {string }}\right)^{-1}
$$

or

$$
\Delta S_{\text {vortex }}=\frac{1}{4 \pi}(\beta \hbar)^{2} T_{\text {string }}\left(1-4 G T_{\text {string }}\right)^{-1}
$$

By combining eq. (4.36) and eq. (4.18), we find the leading charge-dependent contribution to the mass of a black hole of given temperature. Since we also know that the leading charge-independent contribution to the mass is $M \simeq \beta \hbar / 8 \pi G$, we can invert eq. (4.18), and find the charge-dependent correction to the temperature of a black hole of given mass. The result is

$$
\begin{gathered}
\beta^{-1}(M, Q)-\beta^{-1}(M, Q=0) \simeq \\
-\frac{\hbar \eta T_{\text {string }}}{2 \pi M} C(8 \pi G M)\left[1-\cos \left(\frac{2 \pi Q}{N \hbar e}\right)\right] \exp \left(-16 \eta \pi(G M)^{2} T_{\text {string }} / \hbar\right),
\end{gathered}
$$

where we have defined

$$
\eta=\left(1-4 G T_{\text {string }}\right)^{-1}
$$

Thus, the $Z_{N}$ electric charge on a black hole lowers its temperature compared to the temperature of an uncharged hole with the same mass. This effect has the same sign as the effect of unscreened electric charge, in the case of the ReissnerNordström black hole.

\subsection{The Thick String Limit}

We now consider the opposite limit, in which the natural string thickness is large compared to the size of the black hole. In this limit, the action of the vortex can be expanded in powers of $\left(\mu r_{+}\right)^{2}$. The leading term in this expansion can be obtained by setting $v^{2}=0$ in eq. (4.22). Then the action is minimized by setting $\rho=0$ everywhere, and the equations to be solved become identical to those for the 
purely electromagnetic case, which we have already analyzed in Section 3.5. Thus, the vortex action is given by eq. (3.40), with $\omega=2 \pi / N$. We therefore have

$$
\Delta S_{\mathrm{vortex}}=\frac{\pi}{2(N e)^{2}}\left[1+\frac{1}{2} \frac{G}{(\beta \hbar)^{2}}\left(\frac{2 \pi}{N e}\right)^{2}\right] .
$$

In the limit $\hbar \rightarrow 0$ and $v^{2} \rightarrow 0$, the leading charge-dependent contribution to the mass of a black hole of given temperature is found by combining eq. (4.39) with eq. (4.18). We may invert this relation, as before, to find the charge-dependent correction to the temperature of a black hole with given mass. The result is

$$
\begin{aligned}
\beta^{-1}(M, Q) & -\beta^{-1}(M, Q=0) \simeq \\
\frac{\hbar}{2048 \pi G^{3} M^{5}(N e)^{4}} & C(8 \pi G M)\left[1-\cos \left(\frac{2 \pi Q}{N \hbar e}\right)\right] \\
& \times \exp \left\{-\frac{\pi}{2(N e)^{2}}\left[1+\frac{1}{32 G M^{2}(N e)^{2}}\right]\right\} .
\end{aligned}
$$

We see that, in the thick string limit, adding charge to a black hole of fixed mass actually causes the black hole to heat up. This behavior is the opposite of that found in the thin string limit or in the Reissner-Nordström case.

The leading $v^{2}$-dependent correction to $\Delta S_{\text {vortex }}$ is of order $v^{2} r_{+}^{2}=$ $\left(1 /(N e)^{2}\right)\left(\mu r_{+}\right)^{2}$, and so is small compared to the expression in eq. (4.39) provided $\mu r_{+}<<1$, which is just the condition for the natural string thickness to be much larger than the size of the black hole. It is instructive to compare eq. (4.39) with eq. (4.36), which was derived under the assumption $\mu r_{+}>>1$. Suppose that the back reaction of the matter fields on the geometry is a small effect, so that the background geometry of the vortex is very close to the Euclidean Schwarzschild geometry; this is true if

$$
G T_{\text {string }}<<1
$$

in the thin string limit, or if

$$
\frac{G}{(\beta \hbar)^{2}}\left(\frac{2 \pi}{N e}\right)^{2}<<1
$$


in the thick string limit. We then have

$$
\Delta S_{\text {vortex }}^{(\text {thin })} \sim 2 \pi v^{2} r_{+}^{2}
$$

(taking $\left.T_{\text {string }} \sim(1 / 4) v^{2}\right)$, and

$$
\Delta S_{\text {vortex }}^{(t h i c k)} \simeq \frac{\pi}{2(N e)^{2}}
$$

Thus, the thin-string and thick-string expressions for the vortex action cross when $r_{+} \sim \mu^{-1}=(N e v)^{-1}$, as one would expect.

We note that eq. (4.44) has a simple interpretation. Recall that the vortex may be interpreted as a virtual string world sheet that envelops the black hole. Because the string has a finite thickness of order $\mu^{-1}$, the action of a string world sheet has a nonzero minimum value. In flat space, this minimum is of order

$$
S_{\text {world sheet }} \sim 4 \pi \mu^{-2} T_{\text {string }} \sim \frac{\pi}{(N e)^{2}}
$$

This agrees reasonably well with eq. (4.44).

\subsection{The EleCtric Field}

We emphasized in Section 2 that the $Z_{N}$ electric charge on a black hole can be measured by means of the Aharonov-Bohm interaction of the hole with a cosmic string. Now we have seen that an observer who is not equipped with a cosmic string can also detect the charge, by measuring both the mass and the temperature of the black hole radiation.

Even this is not the whole story. Although the black hole has no classical hair, various local observables acquire charge-dependent expectation values due to quantum effects. In particular, there is an electric field outside the event horizon; it could be detected by an experimenter armed with electroscopes and pith balls. 
To understand the origin of this electric field, consider the virtual process in which a loop of cosmic string nucleates at a point on the event horizon of a black hole, sweeps around the horizon two-sphere, then shrinks and annihilates at the antipodal point. The virtual string has magnetic flux in its core; hence its motion creates an electric field orthogonal to the magnetic field and the direction of motion, an electric field in the radial direction. The time-integrated value of this radial field is purely geometrical - although the electric field is proportional to velocity, the time that the string spends at any point on the sphere is inversely proportional to velocity.

We must also average over all possible points of nucleation. This averaging cancels the magnetic field of the string, but the radial electric field survives. We now recognize that our vortex solution, which is rotationally invariant and has nonzero $F_{r \tau}$, represents this averaged string world sheet.

Now we add together the contributions from the sectors with vorticity $k= \pm 1$, which correspond to the two possible orientations of the virtual string. String world sheets of opposite orientation generate electric fields of opposite sign; their contributions would cancel if the two sectors were weighted equally. But if the $Z_{N}$ charge on the black hole is nonzero, then the $k= \pm 1$ sectors are weighted by unequal Aharonov-Bohm phases, and the electric field acquires a non-trivial expectation value.

The calculation of the electric field is greatly simplified if we assume that eq. (4.41) and eq. (4.42) are satisfied; then it is a good approximation to neglect the back reaction of the vortex on the geometry. Because the solutions with $k=0$ and $k= \pm 1$ have the same geometry, we can easily compute the expectation value of a local observable at a fixed spacetime point in the vicinity of the black hole. If we do not make this approximation, then the computation of expectation values of observables is a much more complicated and delicate task.

In each charge sector, we compute the (Euclidean) expectation value of an 
observable $\mathcal{O}$ as

$$
\langle\mathcal{O}\rangle_{\beta, Q}^{(\mathrm{Euc})}=\frac{1}{Z(\beta, Q)} \frac{1}{N} \sum_{k=-\infty}^{\infty} e^{-2 \pi i k Q / N \hbar e} \int_{\beta \hbar, k} \mathcal{O} e^{-S_{E} / \hbar},
$$

where the path integral is restricted to configurations with vorticity $k$, and $Z(\beta, Q)$ denotes the partition function in the sector with charge $Q$. Lorentzian expectation values may then be obtained by continuing to real time.

Applying this formula to the electric field in the black hole sector, we find, in the semiclassical limit,

$$
\left\langle F_{r \tau}\right\rangle_{\beta, Q}^{(\mathrm{Euc})}=-i \sin \left(\frac{2 \pi Q}{N \hbar e}\right) C(\beta \hbar) e^{-\Delta S_{\mathrm{vortex}} / \hbar}\left(F_{r \tau}\right)_{\mathrm{vortex}},
$$

where $(F)_{\text {vortex }}$ denotes $F$ in the $k=1$ vortex solution. By continuing to real time, we find the expectation value of the radial electric field,

$$
\langle E(r)\rangle_{\beta, Q}=\sin \left(\frac{2 \pi Q}{N \hbar e}\right) C(\beta \hbar) e^{-\Delta S_{\text {vortex }} / \hbar}\left(F_{r \tau}(r)\right)_{\text {vortex }} .
$$

If we neglect back reaction, then we may find $(F)_{\text {vortex }}$ by solving the matter field equations on the Euclidean Schwarzschild background geometry. In the thick string limit, the electric field of the vortex is well-approximated, for $r<<\mu^{-1}$, by the field of the Euclidean Reissner-Nordström solution. We therefore have

$$
\left(F_{r \tau}(r)\right)_{\mathrm{vortex}} \simeq\left(\frac{1}{2 N e}\right) \frac{1}{r^{2}}, \quad r<<\mu^{-1} .
$$

We can also find the asymptotic large- $r$ behavior of the electric field by solving the field equations perturbatively in $1 / r$, without making any assumption about whether $\mu(2 G M)$ is large or small. The result is

$$
\left(F_{r \tau}(r)\right)_{\text {vortex }} \simeq C\left(\frac{1}{2 N e}\right)\left(\frac{\mu}{r}+\frac{1}{r^{2}}\right)\left(\frac{2 G M}{r}\right)^{\mu G M} e^{-\mu r}, \quad r>>\mu^{-1}, G M,
$$

where $C$ is a numerical constant of order one, and corrections down by further powers of $G M / r$ have been neglected. Thus we have found that the expectation 
value of the electric field strength outside the horizon is nonvanishing for $Q \neq 0$, and that the field decays exponentially far away from the black hole.

Similarly, we can compute, in the leading semiclassical approximation, the expectation values of all powers of the electric field, obtaining

$$
\begin{aligned}
& \left\langle E(r)^{n}\right\rangle_{\beta, Q}=\text { charge }- \text { independent } \\
& +(-1)^{n / 2} \cos \left(\frac{2 \pi Q}{N \hbar e}\right) C(\beta \hbar) e^{-\Delta S_{\text {vortex }} / \hbar}\left(F_{r \tau}(r)^{n}\right)_{\text {vortex }} \quad(n \text { even }) \\
& \left\langle E(r)^{n}\right\rangle_{\beta, Q}= \\
& (-1)^{(n-1) / 2} \sin \left(\frac{2 \pi Q}{N \hbar e}\right) C(\beta \hbar) e^{-\Delta S_{\text {vortex }} / \hbar}\left(F_{r \tau}(r)^{n}\right)_{\text {vortex }} \quad(n \text { odd }) .
\end{aligned}
$$

We emphasize that the leading contributions to the expectation values of even powers of $E$ are charge-independent. They are just the effects of the ordinary vacuum fluctuations of the free electromagnetic field (in a gravitational background), or, expressed in path-integral language, the effects of one-loop corrections in the $k=0$ sector. The charge-dependent corrections to these are exponentially small additions; thus there is no need for them to be nonnegative, and they are not.

Eqs. (4.51) and (4.52) do not describe the moments of a probability distribution in which an exponentially small background field is added to the usual distribution of vacuum fluctuations. Rather, exponentially rare events (in no one of which the field is extraordinarily small) are added to the usual distribution. This is typical of tunneling processes. Consider the measurement of the total energy in some region near an alpha-unstable nucleus. Most of the time, all one detects are vacuum fluctuations, but every once in an exponentially rare while an alpha particle comes by.

If we attempt to construct $\rho(E)$, the probability distribution whose moments match the expectation values (4.51) and (4.52), we encounter an apparent paradox. 
The obvious answer is

$$
\rho(E)=\text { charge }- \text { independent }+a \delta\left(E-E_{0}\right)+a^{*} \delta\left(E-E_{0}^{*}\right)
$$

where

$$
a=\frac{1}{2} C(\beta \hbar) e^{-\Delta S_{\text {vortex }} / \hbar} e^{-2 \pi i Q / N \hbar e}, \quad \text { and } \quad E_{0}=i\left(F_{r \tau}(r)\right)_{\text {vortex }} .
$$

But this is preposterous; a probability distribution built of delta-functions with imaginary support is obvious nonsense.

We can discover the sense hiding behind this nonsense if we remember the dependence on $\hbar$ of the semiclassical approximation. In leading semiclassical approximation, the probability distribution for the electric field at a given point (or indeed for any dynamical variable) is of the form

$$
\rho(E)=\sum_{i} A_{i}(E) e^{B_{i}(E) / \hbar}
$$

where the $B^{\prime} s$ are independent of $\hbar$ and the $A$ 's are monomials in $\hbar$. In our case, there are three terms in this series. One is the leading one; the others are exponentially suppressed, but are still retained because they are the leading chargedependent terms. If one of the $B$ 's is a real function with a maximum on the real axis, the corresponding term in $\rho$ simulates a delta-function in the small- $\hbar$ limit. However, even if $B$ is a complex function with a complex stationary point, this still simulates a delta-function (with complex support) provided the integral of interest has sufficient analyticity that the contour of integration can be distorted through the stationary point. An example is a Gaussian with complex center, $B_{i}=-\frac{1}{2}(E-z)^{2}$. The contribution of this term to $\left\langle E^{n}\right\rangle$ is proportional to $z^{n}$, for arbitrary complex $z$.

Of course, we don't know the detailed shape of $B(E)$ from our computations. All we know is $E_{0}$ and $B\left(E_{0}\right)$. (Even if we had computed $C(\beta \hbar)$ all it would have 
told us is the value of $A\left(E_{0}\right)\left|B^{\prime \prime}\left(E_{0}\right)\right|^{-1 / 2}$.) Nevertheless, the general situation is clear. The charge-dependent term in $\rho(E)$ acts in the small- $\hbar$ limit like a sum of delta-functions with imaginary support, but in fact, it is nothing of the kind; it is something like a Gaussian with complex center, a very rapidly oscillating function with very many very closely spaced maxima and minima all along the real axis.

\subsection{The Vortex And the No-Hair Theorem}

In the context of the abelian Higgs model that we have been analyzing in this section, the no-hair theorem states the following: ${ }^{[42]}$ If $v^{2}>0$, then any stationary (Lorentzian) black hole solution, such that all gauge-invariant observables are non-singular both at the horizon and at spatial infinity, must have a vanishing electromagnetic field, and a covariantly constant Higgs field, outside the event horizon.

The vortex solution that we have exhibited here demonstrates that there is no corresponding statement about Euclidean black holes. The vortex is stationary in the sense that gauge-invariant quantities are $\tau$-independent, and it is analytic on the minimal "horizon" two sphere at the origin of the $r-\tau$ plane. Thus, there do exist non-singular stationary solutions to the imaginary time field equations that have topology $R^{2} \times S^{2}$ and are asymptotically flat, with a nontrivial $F_{r \tau}$ and an $r$-dependent $|\phi|^{2}$.

The existence of the Euclidean vortex solution is consistent with the no-hair theorem, because when the vortex is continued back to real time, it fails to satisfy the hypotheses of the no-hair theorem. For example, the electric field is imaginary.

We also note that, in a certain sense, the Euclidean vortex is not static. Although gauge-invariant local observables are independent of $\tau$, there is no nonsingular gauge in which the Higgs field $\phi$ is $\tau$-independent, since the phase of the Higgs field advances by $-2 \pi k$ as $\tau$ increases by $\beta \hbar$. The vorticity $k$ is a global property of the solution that changes sign under the transformation $\tau \rightarrow-\tau$. Correspondingly, in any nonsingular gauge, if we perform the naive continuation to 
real time, the gauge-invariant quantity $|\phi|^{2}$ becomes time dependent.

Expectation values of observables on the black hole background are obtained by summing contributions from the various vorticity sectors, as described in Section 4.5. These expectation values are static, and have suitable reality properties, when continued to real time. In accord with the no-hair theorem, though, the expectation values do not solve the classical field equations; since the field equations are nonlinear, a sum of solutions is not a solution. In short, by performing the sum over $k$, we proceed from non-static solutions to static non-solutions, thus violating the spirit of the no-hair theorem while respecting its mathematical content.

We should also remark that the status of the no-hair theorem for the abelian Higgs system remains unsettled, as was recently stressed by Gibbons. ${ }^{[43]}$ The (classical) no-hair property seems very plausible, but no fully satisfying and sufficiently general proof has yet been found.

We conclude this discussion with some further remarks concerning the $\mu \rightarrow 0$ limit. For classical Lorentzian black holes, this limit is highly singular. If $\mu=0$, then a stationary black hole can have an electric field. But for any nonzero $\mu-$ however small-no electric field is allowed.

One might hope that black hole physics will behave more smoothly in the $\mu \rightarrow 0$ limit, when the effects of quantum hair are properly taken into account. Indeed, for Euclidean solutions, as we have seen, the situation is different. Our vortex solution smoothly approaches the corresponding solution (with the same value of $\omega=2 \pi k / N)$ for a black hole coupled to massless electrodynamics.

Nevertheless, the discontinuous behavior of black hole physics in the $\mu \rightarrow 0$ limit persists. We have found that the expectation value of the electric field outside a black hole that carries $Z_{N}$ charge, while nonvanishing, is far from classical. It is, in fact, exponentially small for small $\hbar$. In contrast, if $\mu=0$, the expectation value is $\hbar$-independent.

This discontinuous behavior seems less mysterious, though, when we recognize that the semiclassical limit has been taken in a much different way for a black 
hole with $Z_{N}$ charge than for a black hole with ordinary electric charge, as we emphasized in Section 4.2. A black hole with specified $Z_{N}$ charge has fixed $Q / N \hbar e$, and so the electric charge $Q$ is of order $\hbar$. We can hardly expect this object to behave like a black hole with a specified classical charge, in the limit $\hbar \rightarrow 0$.

If we want the semiclassical physics of a black hole with quantum hair to behave smoothly in the $\mu \rightarrow 0$ limit, then, we must define the semiclassical limit differently than before. In particular, we should allow $\mathrm{N}$ to become large; then the sum in eq. (4.2) approximates the integral in eq. (3.14). However, there is a catch. To justify replacing the sum in eq. (4.2) by an integral as $N \rightarrow \infty$, we require

$$
Z(\beta, k+1)-Z(\beta, k) \rightarrow 0
$$

But, for $v^{2} \rightarrow 0$ (and neglecting gravitational back reaction), we have

$$
Z(\beta, k) \simeq \exp \left(-\Delta S_{k}^{(t h i c k)}\right) \simeq \exp \left(-\frac{\pi k^{2}}{2 \hbar(N e)^{2}}\right)
$$

So $Z(\beta, Q)$ in eq. (4.2) will not be well approximated by the corresponding expression in massless electrodynamics unless

$$
N \hbar e>>, \quad \hbar(N e)^{2}>>1
$$

The catch is that $\hbar(N e)^{2}$ is a loop expansion parameter. Thus, if eq. (4.58) is satisfied, the Higgs model is strongly coupled, and the semiclassical evaluation of $Z(\beta, k)$ cannot be justified.

The conclusion is that, while it is conceivable that, in a suitable limit, the physics of a black hole with screened electric hair can match smoothly with the physics of a black hole with unscreened hair, this cannot happen within the domain of validity of the semiclassical approximation.

In the next section, we will find that, in the case of magnetic quantum hair, the situation is quite different. 


\section{Screened Magnetic Charge}

\section{1. $Z_{N}$ Magnetic Charge Projection}

As we have already noted in Sections 2 and 3, a gauge theory with an unbroken $S U(N) / Z_{N}$ gauge symmetry can contain magnetic monopoles with $Z_{N}$ magnetic charges. In the classical approximation, such a monopole has an infinite range magnetic field. But if $S U(N) / Z_{N}$ is not spontaneously broken, we expect that, due to quantum effects, the vacuum of the theory is magnetically disordered. Hence the theory exhibits color confinement - electric fields are confined to stable flux tubes, and magnetic fields are screened. The magnetic field of a monopole decays like $e^{-\mu r}$ at long range, where $\hbar \mu$ is the glueball mass gap.

Nevertheless, in spite of the magnetic screening, the $Z_{N}$ magnetic charge of an object can be detected in principle at arbitrarily long range, by means of the nontrivial Aharonov-Bohm interaction of a magnetic charge with an electric flux tube. It follows that, despite the screening, a stationary black hole can carry magnetic charge. ${ }^{[23]}$ Our objective in this section is to perform a semiclassical analysis of the effect of $Z_{N}$ magnetic charge on the physics of a black hole..$^{[2,26]}$

Evidently, the detection of $Z_{N}$ magnetic charge with an electric flux tube is strikingly similar to the detection of $Z_{N}$ electric charge with a cosmic string. Since we have already analyzed the effects of $Z_{N}$ electric charge on a black hole in much detail, one may wonder whether it is really necessary to repeat the whole analysis for the case of magnetic charge. In fact, we will find that the semiclassical theory of a black hole with $Z_{N}$ magnetic charge is considerably different than the theory that we have already developed for a black hole with $Z_{N}$ electric charge. The origin of the difference is already noted in the preceding discussion: While the screening of electric fields due to the Higgs mechanism is a classical phenomenon (zeroth order in $\hbar$ ), the screening of magnetic field due to confinement is a quantum effect (actually nonperturbative in $\hbar$ ).

To calculate the effect of $Z_{N}$ magnetic charge on black hole thermodynam- 
ics, we must first devise a Euclidean path integral prescription for computing the partition function restricted to a particular magnetic charge sector. For the case of $U(1)$ magnetic charge discussed in Section 3.7, we saw that a magnetic charge projection was implemented trivially - the sector with magnetic charge $P$ is obtained by restricting the path integral to configurations with magnetic flux $4 \pi P$ at $r=\infty$. In spite of the screening, a similar procedure applies in the case of $Z_{N}$ magnetic charge. As explained in Section 2.4, the total magnetic charge can be characterized by the topology of the gauge field (or, alternatively, the Higgs field) on the two-sphere at $r=\infty$. The charge projection is performed by restricting the field configurations to the appropriate topological class.

\subsection{Electric-Magnetic Duality}

Because of the strong similarity between $Z_{N}$ electric and $Z_{N}$ magnetic charge, it seems odd that the charge projections in the two cases are so different. We saw that the expression eq. (4.2) for $Z(\beta, Q)$ has a very appealing interpretation. We may think of the vorticity $k$ as the number of times that a virtual cosmic string world sheet wraps around the horizon of the black hole, and the phase multiplying $Z(\beta, k)$ is just the Aharonov-Bohm phase acquired by this virtual string. Surely, we should be able to understand the dependence of black hole thermodynamics on the $Z_{N}$ magnetic charge in a similar way, for the effect of the magnetic charge is to weight the contributions due to virtual electric flux tubes by appropriate Aharonov-Bohm phases. One suspects, therefore, that there is an alternative way to construct the $Z_{N}$ magnetic charge projection that is more closely parallel to what we did for $Z_{N}$ electric charge.

This is indeed the case, as we can see by performing an electric-magnetic duality transformation. ${ }^{[4]}$ In an $S U(N)$ gauge theory with an unbroken local $Z_{N}$ symmetry, states can be classified according to how they transform under $Z_{N}$. The $Z_{N}$ electric charge $\tilde{e}$ is defined by

$$
U(\tilde{m})|\tilde{e}\rangle=e^{2 \pi i \tilde{m} \tilde{e} / N}|\tilde{e}\rangle
$$


where $U(\tilde{m})$ represents the $Z_{N}$ transformation $e^{2 \pi i \tilde{m} / N}$; this charge takes the values

$$
\tilde{e}=0,1,2, \ldots, N-1
$$

We may insert the projection operator

$$
P(\tilde{e})=\frac{1}{N} \sum_{\tilde{m}=0}^{N-1} e^{-2 \pi i \tilde{m} \tilde{e} / N} U(\tilde{m})
$$

into the path integral, as in Sections 3.3 and 4.1, to obtain an expression for the partition function in the sector with charge $\tilde{e}$; the result is

$$
Z(\beta, \tilde{e})=\frac{1}{N} \sum_{\tilde{m}=0}^{N-1} e^{-2 \pi i \tilde{m} \tilde{e} / N} Z(\beta, \tilde{m})
$$

where $Z(\beta, \tilde{m})$ is a path integral over configurations that satisfy the constraint

$$
\left[P \exp \left(i e \int_{0}^{\beta \hbar} d \tau A_{\tau}(\tau, \vec{x})\right)\right]_{r=\infty}=e^{2 \pi i \tilde{m} / N}
$$

In the black hole sector, with topology $R^{2} \times S^{2}$, we may think of $\tilde{m}$ as the topological magnetic flux, or vorticity, in the $r-\tau$ plane. Naturally, we can invert the Fourier transform and write

$$
Z(\beta, \tilde{m})=\sum_{\tilde{e}=0}^{N-1} e^{2 \pi i \tilde{m} \tilde{e} / N} Z(\beta, \tilde{e})
$$

Now consider the case of an $S U(N) / Z_{N}$ gauge theory that contains no dynamical $Z_{N}$ electric or magnetic charges. Then we may think of $\tilde{e}$ as the electric flux on each $S^{2}$ parametrized by $\theta$ and $\phi$. And imagine that the $r-\tau$ plane is compactified to a two-sphere, so that the Euclidean black hole geometry has the 
topology $S^{2} \times S^{2}$. The way to derive a duality relation is to interchange the two two-spheres,

$$
S^{2}(\theta, \phi) \longleftrightarrow S^{2}(r, \tau)
$$

In other words, we may reinterpret eq. (5.6), regarding $\tilde{m}$ as the magnetic flux on $S^{2}(\theta, \phi)$, and $\tilde{e}$ as the electric flux on $S^{2}(r, \tau)$. So, at least in the case where there are no dynamical magnetic monopoles, the partition function of a black hole with specified $Z_{N}$ magnetic charge $\tilde{m}$ is obtained by summing the sectors with various values of the electric vorticity $\tilde{e}$, weighted by appropriate phases. The electric vorticity is the number of times that a virtual electric flux tube wraps around the black hole horizon, and the associated phase is just the Aharonov-Bohm phase acquired by the virtual flux tube. This is the result we expected.

\subsection{The Thin String Limit}

In a confining gauge theory, the thickness $\mu^{-1}$ and tension $T_{\text {string }}$ of an electric flux tube are, in order of magnitude,

$$
\mu^{-1} \sim \frac{\hbar}{\Lambda}, \quad T_{\text {string }} \sim \frac{\Lambda^{2}}{\hbar}
$$

where $\Lambda$ is the characteristic mass scale of the theory. Of course, the tension and mass gap are really nonperturbative in $\hbar$; we may express the mass scale $\Lambda$ as

$$
\frac{\Lambda}{\hbar} \sim a^{-1} \exp \left[-\frac{1}{b \hbar e^{2}(a)}\right]
$$

where $a$ is a short-distance cutoff, and $e^{2}(a)$ is the bare gauge coupling. There is no confinement, and no magnetic screening (at zero temperature), to any finite order in the $\hbar$ expansion.

Now consider the effect of $Z_{N}$ magnetic charge on a black hole, in the case where the screening length $\mu^{-1}$ is very small compared to the size $r_{+}$of the black hole (the "thin string limit"). The classical black hole solution has a long-range 
magnetic field, but the classical approximation is badly misleading in the thin string limit; nonperturbative effects screen the magnetic field, and nearly extinguish it entirely.

The leading charge-dependent effects in the thin string limit are due to a virtual electric flux tube that wraps once around the event horizon, with either orientation. These effects are most easily analyzed, then, using the dual formulation described in the previous section; the $\tilde{e}= \pm 1$ sectors dominate the charge-dependence in eq. (5.6). Of course, since nonperturbative effects are important, the evaluation of the charge dependence of the free energy is not, strictly speaking, semiclassical. But if we imagine working with an effective field theory that describes the infrared behavior of the confining theory, then the analysis becomes virtually identical to that described in Section 4.3.

For $\mu r_{+}>>1$, the path integral in the sectors with $\tilde{e}= \pm 1$ is dominated by a configuration with a pointlike electric vortex sitting at the origin of the $r-\tau$ plane. In this configuration, the effective matter field action is

$$
S_{\text {matter }}^{(e f f)} \simeq 4 \pi r_{+}^{2} T_{\text {string }}
$$

as in eq. (4.26). Proceeding as in Section 4.3, we find the leading charge-dependent contribution to the black hole temperature,

$$
\beta^{-1}(M, \tilde{m})-\beta^{-1}(M, \tilde{m}) \propto\left[1-\cos \left(\frac{2 \pi \tilde{m}}{N}\right)\right] \exp \left(-16 \pi(G M)^{2} T_{\text {string }} / \hbar\right)
$$

(for $G T_{\text {string }}<<1$ ).

The virtual electric flux tubes that sweep around the black hole generate a radial magnetic field outside the horizon, as in the discussion in Section 4.5. Of course, $F_{\theta, \phi}$ is not gauge invariant in a nonabelian theory, but we can characterize the field by considering, for example, the expectation value of the gauge-invariant 
operator $\operatorname{tr}\left(F_{\theta, \phi}^{2}\right)$. This behaves as

$$
\begin{aligned}
& \left\langle\operatorname{tr} F_{\theta, \phi}(r)^{2}\right\rangle_{M, \tilde{m}} \sim \text { charge independent } \\
& \quad+\cos \left(\frac{2 \pi \tilde{m}}{N}\right) \exp \left(-16 \pi(G M)^{2} T_{\text {string }} / \hbar\right) e^{-2 \mu r} .
\end{aligned}
$$

The charge-dependent field is suppressed not just by the screening factor $e^{-2 \mu r}$, but also by the tunneling factor $\exp \left(-\Delta S_{\text {vortex }}^{(e f f)} / \hbar\right)$. Notice that its dependence on $\tilde{m}$ is different than that of the classical solution described in Section 3.8; it is proportional to $\cos (2 \pi \tilde{m} / N)$ instead of $\tilde{m}(N-\tilde{m})$.

\subsection{The Thick String Limit}

Now we consider the opposite limit, in which the thickness $\mu^{-1}$ of an electric flux tube is much larger than the size $r_{+}$of the black hole. In this limit, it is more convenient to implement the magnetic charge projection by restricting the path integral to configurations with specified $Z_{N}$ flux $\tilde{m}$. Then the evaluation of the black hole partition function, in the leading semiclassical approximation, proceeds much as in the magnetic Reissner-Nordström case discussed in Section 3.7. The path integral is dominated by the magnetically charged black hole classical solution that we described in Section 3.8 (or, if that solution is unstable, by a solution analogous to the one constructed in Ref. 36).

Of course, we are obligated to consider the nonperturbative corrections. In the thin string limit, these completely invalidate the leading semiclassical result, as we saw above. But in the thick string limit, the nonperturbative corrections to the semiclassical calculation are small. The Yang-Mills action of the magnetically charged black hole is dominated by the magnetic field close to the event horizon. In the thick string limit, asymptotic freedom ensures that the gauge coupling renormalized at the distance scale $r_{+}$is small; hence, quantum fluctuations about the classical solution are suppressed near the horizon. Nonperturbative quantum cor-

rections are important at a distance of order $\mu^{-1}$ from the black hole, where the 
magnetic field begins to become screened. But the nonperturbative contribution to the Yang-Mills effective action is suppressed, relative to the classical contribution, by a factor of order $\left(\mu r_{+}\right)^{2}$, and so can be neglected in the thick string limit.

Therefore, in the thick-string limit, it is a good approximation to saturate the path integral with the appropriate Euclidean classical solution. For values of the parameters such that the the solution described in Section 3.8 is stable, then, the thermodynamics of a black hole with $Z_{N}$ magnetic charge $\tilde{m}$, in the thick string limit and the leading semiclassical approximation, is identical to that of a Reissner-Nordström black hole, where the effective charge $P_{\tilde{m}}$ of the hole is

$$
P_{\tilde{m}}^{2}=\left(\frac{1}{2 e^{2}}\right) \frac{\tilde{m}(N-\tilde{m})}{N}
$$

(as in eq. (3.59); $e^{2}$ denotes the gauge coupling renormalized at distance scale $r_{+}$). The effect of the charge on the thermodynamics can be quite significant; it can even lower the temperature to zero and shut down the Hawking evaporation of the hole. Furthermore, outside the event horizon, and for $r<<\mu^{-1}$, the black hole has a magnetic field that is well approximated by that of the classical solution.

\subsection{Screened Magnetic Charge vs. Screened Electric Charge}

As we have seen, the physical effects of screened electric and magnetic charge on a black hole are similar in the thin string limit, but dramatically different in the thick string limit. Why?

The difference arises because of the different role played by $\hbar$ in the two cases. The electric charge quantum is of order $\hbar e$. So $Z_{N}$ electric charge is really quantum-mechanical, and becomes irrelevant in the classical limit. But the magnetic charge quantum is of order $1 / e$. So $Z_{N}$ magnetic charge can have effects that survive in the classical limit.

Furthermore, since the magnetic flux carried by a cosmic string is of order $1 / e$, the cosmic string is a classical object, and virtual strings arise as quantummechanical fluctuations only very rarely. In contrast, an electric flux tube carries a 
flux of order $\hbar e$, and virtual strings occur copiously as quantum fluctuations. The dependence of black hole physics on $Z_{N}$ electric charge is dominated by configurations such that a single string world sheet wraps around the black hole, even if the string is very thick. But, because the electric flux tube carries a small flux, the cost in effective action of adding another flux tube, in the thick string limit, is correspondingly small. Thus, the dependence on the $Z_{N}$ magnetic charge is dominated by configurations that contain not a single string, but many. We may think of the classical magnetic field as resulting from the "condensation" of the cloud of virtual strings that surrounds the black hole.

Since an electric flux tube is really a nonperturbative object, it may seem miraculous that the effects of virtual electric flux tubes admit a classical description. Asymptotic freedom makes this miracle possible. The effective action of the cloud of virtual strings surrounding the hole is dominated by the fields close to the event horizon, where they may be regarded as weakly coupled.

The sharp distinction between screened electric fields and screened magnetic fields applies if we insist on analyzing both in the semiclassical approximation. A confining theory that is weakly coupled at the event horizon of a black hole exhibits much different physics than a Higgs theory that is weakly coupled at the event horizon. But the distinction blurs as the confinement length scale becomes comparable to the size of the hole, so that virtual electric flux tubes that envelop the hole become suppressed, or if the Higgs theory is strongly coupled, so that virtual cosmic strings are unsuppressed.

Our analysis of screened magnetic charge enables us to reexamine an issue that we previously addressed in Section 4.6: Is it possible for the physics of a black hole with screened hair to match up smoothly, as the inverse screening length $\mu$ approaches zero, with the physics of a black hole with unscreened hair? In the case of $Z_{N}$ electric hair, we saw that this is not possible if the Higgs theory is weakly coupled. Insofar as a strongly-coupled abelian Higgs theory resembles a weakly-coupled nonabelian theory, it seems appropriate to reopen the question 
now.

Suppose that we introduce into an $S U(N) / Z_{N}$ gauge theory the necessary Higgs structure so that, by appropriately adjusting the parameters of the Higgs potential, we may break the gauge group to the abelian subgroup $U(1)^{N-1}$. In this "Coulomb phase" of the model, there are black hole solutions that carry $U(1)^{N-1}$ charges. Among these solutions are ones that have the same magnetic field as the black hole solutions of a model with unbroken $S U(N) / Z_{N}$, the solutions that we described in Section 3.8. But, in the Coulomb phase there is no confinement and no magnetic screening; the classical magnetic field survives at arbitrarily long range.

Now, in the semiclassical approximation, the black hole partition function in a sector with specified magnetic charge is dominated by the corresponding magnetically-charged solution. This is true in both the Coulomb phase and the confining phase of the model. Furthermore, the black hole solutions in the Coulomb phase have a covariantly constant Higgs field. (The instability described in Ref. 36 does not apply here. The Coulomb field extends all the way into the horizon whether or not the Higgs field that breaks $S U(N) / Z_{N}$ to $U(1)^{N-1}$ turns on outside the horizon.) Thus, the leading semiclassical thermodynamics of a magneticallycharged black hole in the confining phase is identical to the leading semiclassical thermodynamics of a charged black hole in the Coulomb phase.

If the Higgs symmetry breaking scale $v$ is small, so that effects that depend on the details of the Higgs potential may be neglected, the semiclassical expansion of the partition function is the same, to each order in $\hbar$, in the Coulomb phase as in the confining phase. Finally, we recall that nonperturbative corrections in the confining phase are small when the confinement length scale is much larger than the black hole. We conclude that the physics of a magnetically charged black hole in the confining phase, in the limit $\mu r_{+}<<1$, coincides with the physics of a magnetically charged black hole in the Coulomb phase, in the limit evr $r_{+}<<1$. In this instance, no annoying discontinuity is encountered as the inverse screening length $\mu$ goes to zero. 
Our study of screened electric and magnetic hair on black holes may be summarized as follows: The no-hair theorems assert that classical hair is incompatible with classical screening. But they leave open two possible ways to evade the dictum that a black hole has no (screened) hair-either the hair or the screening (or, conceivably, both) may be quantum mechanical. In a weakly-coupled Higgs system, the screening of electric fields is classical in the sense that the screening length $\mu^{-1}$ is independent of $\hbar$. We have seen that this classical screening can be reconciled with a non-vanishing electric field outside the event horizon of a black hole, provided that the field disappears in the $\hbar \rightarrow 0$ limit. On the other hand, in a confining gauge theory, the screening of magnetic fields is quantum-mechanical in the sense that $\mu \rightarrow 0$ as $\hbar \rightarrow 0$. We have seen that this non-classical screening is compatible with a classical ( $\hbar$-independent) magnetic field outside the horizon.

\section{Dual Formulation of Broken Symmetry Phases}

In this section we shall discuss the representation of Goldstone boson, Stückelberg-Higgs, and axion theories in dual form. The use of such alternative representations can be a source of insight (or confusion) in a wide variety of problems, including but not restricted to black hole physics. We will now work in Lorentzian spacetime, unless otherwise noted.

\subsection{Goldstone Boson}

To begin consider the theory of a massless scalar (Goldstone) field $\phi$, described by the Lagrangian

$$
\mathcal{L}=\frac{F^{2}}{2}\left(\partial_{\mu} \phi\right)^{2}
$$

(For simplicity we shall write our formulas in the form appropriate for flat space. Their generalization to curved space is more or less immediate; see below.) This 
Lagrangian may also be obtained from

$$
\mathcal{L}=i \partial_{\mu} \phi J^{\mu}+\frac{1}{2 F^{2}} J_{\mu}^{2}
$$

by eliminating $J_{\mu}$, which appears only algebraically. Indeed by setting the variation with respect to $J$ equal to zero we find

$$
J_{\mu}=-i F^{2} \partial_{\mu} \phi
$$

and substituting this into (6.2) yields (6.1).

The same equations of motion result if we add a total derivative to the action. Thus we will obtain the same equations of motion if we integrate the first term on the right-hand side of (6.2) by parts, and drop the surface term. This procedure gives us the Lagrangian

$$
\mathcal{L}=-i \phi \partial_{\mu} J^{\mu}+\frac{1}{2 F^{2}} J_{\mu}^{2}
$$

in which $\phi$ appears only algebraically. The variation with respect to $\phi$ simply gives us the constraint

$$
\partial_{\mu} J^{\mu}=0
$$

expressing conservation of the current $J$. The Lagrangian is just

$$
\mathcal{L}=\frac{1}{2 F^{2}} J_{\mu}^{2}
$$

subject, of course, to the constraint (6.5).

In three-plus-one space-time dimensions one may solve the constraint by writing

$$
J^{\mu}=\frac{-i}{2} \epsilon^{\mu \nu \rho \sigma} \partial_{\nu} B_{\rho \sigma}
$$

where $B$ is an antisymmetric tensor field. Substituting this into (6.6), we see that the Goldstone boson theory has been re-written in terms of a two index antisymmetric tensor field. 
In curved space one starts with the Goldstone boson Lagrangian

$$
\mathcal{L}=\frac{F^{2}}{2} \sqrt{g} g^{\mu \nu} \partial_{\mu} \phi \partial_{\nu} \phi
$$

One may obtain this from

$$
\mathcal{L}=i \partial_{\mu} \phi J^{\mu}+\frac{1}{2 F^{2} \sqrt{g}} g_{\mu \nu} J^{\mu} J^{\nu}
$$

In this formulation $J^{\mu}=\sqrt{g} g^{\mu \nu} \partial_{\nu} \phi$ is a vector density which satisfies $\partial_{\mu} J^{\mu}=0$ identically. $B$ may be introduced as before, to solve the constraint; it is a proper two-index tensor (the numerical epsilon symbol is a tensor density, and absorbs the excess $\sqrt{g}$ ).

Let us now briefly consider how this formalism applies to the theory of a complex scalar field. Let $\eta$ be a complex scalar described by the Lagrangian

$$
\mathcal{L}=\left|\partial_{\mu} \eta\right|^{2}-V(|\eta|)
$$

where $V$ is the potential term. Writing $\eta$ in polar form $\eta=\rho e^{i \phi}$ we have

$$
\mathcal{L}=\left(\partial_{\mu} \rho\right)^{2}+\rho^{2}\left(\partial_{\mu} \phi\right)^{2}-V(\rho)
$$

Now if $\rho$ develops an expectation value of magnitude $F / \sqrt{2}$, we simply expand $\rho \equiv \frac{1}{\sqrt{2}}(F+\zeta)$. Whereas $\rho$ was constrainted to be $\geq 0$, we have $-F \leq \zeta$. The $\phi$-dependent terms in (6.11) are then simply

$$
\mathcal{L}=\frac{1}{2}(F+\zeta)^{2}\left(\partial_{\mu} \phi\right)^{2}
$$

Now we can introduce the dual description much as before. The Lagrangian will still be of the current-squared form, but the constraint for the dual current will be 
altered, to assume the form

$$
\partial_{\mu}\left(J^{\mu}\left(1+\frac{\zeta}{F}\right)\right)=0
$$

This can be solved in the form

$$
J^{\mu}=\frac{-i}{2} \frac{\epsilon^{\mu \nu \rho \sigma} \partial_{\nu} B_{\rho \sigma}}{(1+\zeta / F)}
$$

This expression becomes singular where $\zeta$ approaches its minimum (i.e. where the magnitude $\rho$ of the original scalar field vanishes).

Thus the dual formulation can be extended to the full complex scalar theory, in a rather straightforward fashion. Also, the larger theory will tell us how to proceed, in principle, at the singular points where $\rho=\frac{1}{\sqrt{2}}(F+\zeta)$ vanishes - such as occurs at the center of cosmic strings, where the earlier formulation breaks down.

\subsection{Gauge Coupling}

The Goldstone boson Lagrangian (6.1) is invariant under the transformation $\phi \rightarrow \phi+\lambda$, where $\lambda$ is a numerical constant. We can promote this to a local symmetry transformation by coupling to a gauge field with appropriate transformation properties. Indeed

$$
\mathcal{L}=\frac{F^{2}}{2}\left(\partial_{\mu} \phi+e A_{\mu}\right)^{2}
$$

is invariant under the local transformation

$$
\begin{gathered}
\phi \rightarrow \phi+e \lambda \\
A_{\mu} \rightarrow A_{\mu}-\partial_{\mu} \lambda
\end{gathered}
$$

where $\lambda$ is a function on space and time. 
The gauge freedom can be used to set $\phi=0$. Then (6.15) reduces to $\frac{1}{2}(e F)^{2} A_{\mu}^{2}$, that is to a mass term for $A$. This is the Higgs mechanism in its simplest form, as first presented by Stückelberg. It is also essentially equivalent to the London theory of superconducting electrodynamics. As we have just seen in the global symmetry case, $\phi$ can be regarded as the phase of an ordinary complex scalar field, that acquires a vacuum expectation value. This explains the additive form of the gauge transformation law (6.16). (The Stückelberg-London Lagrangian is an approximation to the usual Landau-Ginzburg Lagrangian, in which the magnitude of the scalar field is frozen and only its phase allowed to vary.)

To construct the dual form of this theory, we introduce

$$
\mathcal{L}=i J^{\mu}\left(\partial_{\mu} \phi+e A_{\mu}\right)+\frac{1}{2 F^{2}} J_{\mu}^{2}
$$

It is easy to verify that elimination of $J$ from (6.17) leads right back to (6.15). Alternatively one may integrate by parts, and eliminate $\phi$. This leads to the constraint $\partial_{\mu} J^{\mu}=0$ and to the simple Lagrangian

$$
\mathcal{L}=i e J^{\mu} A_{\mu}+\frac{1}{2 F^{2}} J_{\mu}^{2}
$$

with $J_{\mu}$ subject to the conservation constraint.

As before, we may solve the constraint by writing $J$ in the form (6.7) . This leads to the Lagrangian

$$
\mathcal{L}=\frac{e}{2} \epsilon^{\mu \nu \rho \sigma} A_{\mu} \partial_{\nu} B_{\rho \sigma}-\frac{1}{2 F^{2}}\left(\epsilon^{\mu \nu \rho \sigma} \partial_{\nu} B_{\rho \sigma}\right)^{2}
$$

Thus the difference between the Goldstone and the Higgs theories, in this dual form, is simply the absence or presence of the first term on the right-hand side of (6.19) . Indeed this term is just the current-gauge field coupling, written in a slightly unfamiliar form. It may also be written in another, suggestive way after 
an integration by parts:

$$
\frac{e}{2} \epsilon^{\mu \nu \rho \sigma} A_{\mu} \partial_{\nu} B_{\rho \sigma} \rightarrow \frac{e}{4} \epsilon^{\mu \nu \rho \sigma} F_{\mu \nu} B_{\rho \sigma} .
$$

In the first form the potential $A$ of the gauge field occurs, but only the combination $H_{\mu \nu \rho}=\partial_{\mu} B_{\nu \rho}+$ cyclic permutations - i.e., the field strength - for the antisymmetric tensor appears. In the second form the roles are reversed. Only the field strength for the gauge field occurs, but the bare potential $B$ appears. In the second form, the simple charge coupling has come closely to resemble the $\theta$ term of gauge theories. This form of the gauge coupling in the Higgs theory will play a central role in our analysis in the following chapter.

In the next chapter we shall need the curved-space form of the dualized gauge theory. For future reference, then, let us record the generalizations of $(6.17),(6.19)$

$$
\begin{aligned}
& \mathcal{L}=i J^{\mu}\left(\partial_{\mu} \phi+e A_{\mu}\right)+\frac{1}{2 F^{2} \sqrt{g}} g_{\mu \nu} J^{\mu} J^{\nu} \\
& \mathcal{L}=\frac{e}{4} \epsilon^{\mu \nu \rho \sigma} F_{\mu \nu} B_{\rho \sigma}+\frac{1}{2 F^{2} \sqrt{g}} g_{\mu \nu} J^{\mu} J^{\nu} .
\end{aligned}
$$

The contribution of the first term in (6.22) to the action is actually independent of the spacetime metric; it can be expressed in terms of a wedge product of differential forms. The term $S_{B \wedge F}$ that couples the gauge field to the antisymmetric tensor field has the form

$$
e^{i S_{B \wedge F} / \hbar}=\exp \left(\frac{i e}{\hbar} \int B \wedge F\right) .
$$




\subsection{AXION}

As our final example we consider the dual formulation of axion physics. The basic Lagrangian (for an axion coupled to an abelian gauge field) is

$$
\mathcal{L}=\frac{f^{2}}{2}\left(\partial_{\mu} \phi\right)^{2}+\phi \epsilon^{\mu \nu \rho \sigma} F_{\mu \nu} F_{\rho \sigma}
$$

In the now familiar way, we obtain this from the alternative Lagrangian

$$
\mathcal{L}=i J^{\mu} \partial_{\mu} \phi+\frac{1}{2 f^{2}} J_{\mu}^{2}+\phi \epsilon^{\mu \nu \rho \sigma} F_{\mu \nu} F_{\rho \sigma}
$$

by eliminating $J$. Alternatively, integrating by parts and eliminating $\phi$ yields the constraint

$$
\partial_{\mu} J^{\mu}=i \epsilon^{\mu \nu \rho \sigma} F_{\mu \nu} F_{\rho \sigma} .
$$

This constraint may be solved in the form

$$
J^{\mu}=\frac{-i}{2} \epsilon^{\mu \nu \rho \sigma} \partial_{\nu} B_{\rho \sigma}+\frac{i}{2} \epsilon^{\mu \nu \rho \sigma} A_{\nu} F_{\rho \sigma} .
$$

In terms of this constrained $J$, the Lagrangian is simply $\mathcal{L}=\frac{1}{2 f^{2}} J_{\mu}^{2}$. Upon writing out this Lagrangian in terms of the antisymmetric tensor $B$, one finds peculiar cross-couplings between $B$ and the gauge fields, which are not manifestly gauge invariant. They are closely related to the Green-Schwarz terms that play an important role in superstring theory. ${ }^{[45]}$ From the point of view of axions, they reflect

fact that exchange of the axion between photons involves a $\frac{1}{q^{2}}$ propagator which partially cancels the derivatives coming from the $\phi \epsilon^{\mu \nu \rho \sigma} F_{\mu \nu} F_{\rho \sigma}$ vertices.

The gauge transformation property of $B$ is peculiar. It follows from the gauge invariance of $J$ in (6.27). Thus under a gauge transformation we have

$$
\begin{gathered}
B_{\mu \nu} \rightarrow B_{\mu \nu}-\lambda F_{\mu \nu} \\
A_{\mu} \rightarrow A_{\mu}-\partial_{\mu} \lambda .
\end{gathered}
$$




\section{Duality and Hair}

In this chapter we shall look at quantum hair from a different perspective, using the dual formalism developed in the previous chapter. We shall find, of course, that this formalism yields a superficially different but fully equivalent description of the earlier results. The black hole hair will be associated with the fractional part of the surface integral of the two-form $B$ over large spheres surrounding the black hole - in our previous language, this integral is the $Z_{N}$ charge divided by $N$. The $B$ field gives a more classical look to the hair, in that there is a definite long-range field attached to it. ${ }^{[21,46]}$ However this appearance is deceptive: the meaningful part of $B$ is not a classical field in the usual sense. One sign of this, is its observable (fractional) part cannot take arbitrarily large values. Indeed the fractional charge associated with $B$ only acquires physical significance in the interaction of the hole with real or virtual flux tubes - precisely the same process as we have described in great detail above, which is non-perturbative in $\hbar$. Nevertheless the $B$ field is very useful in describing the space-time process of cosmic string lassoing a charged hole, as we shall see..$^{[24,25]}$

The interpretation of " $B$ hair" as gauge hair depends on the presence of the $B \wedge F$ interaction in the Lagrangian, which occurs in the dual theory of a massless scalar precisely when that scalar is eaten by the vector field according to the Higgs mechanism. We emphasize that $B$ hair is therefore, in the only case where we understand it, nothing but discrete gauge hair. In particular it has nothing to do with axions, despite the unfortunate terminology common in the literature. One might also be tempted to consider the possibility of global hair, for a discrete remnant of a broken global symmetry. Global symmetry corresponds to the absence of a $B \wedge F$ term. We will offer both formal and physical arguments against the possibility of meaningful hair in this case, however. ${ }^{[25]}$ 


\subsection{Dual Charge}

Harking back to (6.19), the charge $Q$ that couples to the gauge field can be expressed in terms of the antisymmetric tensor field. The charge contained within a region $\Omega$ is

$$
Q_{\Omega}=\frac{e}{2} \int_{\Omega} \epsilon^{i j k} \partial_{i} B_{j k} d^{3} x=\frac{e}{2} \oint_{\Sigma} \epsilon^{i j k} B_{j k} d^{2} S_{i} \equiv e \int_{\Sigma} B ;
$$

it is ( $e$ times) the integral of the two-form $B$ over the closed surface $\Sigma$, the boundary of $\Omega$.

Now on the black hole geometry one can have a non-zero contribution to the surface integral of a peculiar form. Consider a $B$ field that takes the form

$$
e B=\frac{Q}{4 \pi} \sin \theta d \theta \wedge d \phi
$$

outside the event horizon of the hole. This form is closed, so that the charge density $H=d B$ vanishes outside the black hole. However if we focus on the final entry in (7.1) , an interesting possibility comes into view. That is, there is the possibility, exploited precisely by a $B$ field of the given form, of a contribution from a bounding surface - in our example, the event horizon - which gets cancelled, formally, only by the boundary at infinity. Thus we might expect that the $B$ field can represent charge within the black hole. If so, the charge $Q$ is a type of black hole hair.

However the physical significance of the hair thus defined formally is not immediately obvious. Indeed $B$ was introduced as an auxiliary quantity in the dualization process, and has no immediately given independent meaning. To understand the meaning of this hair, we must consider the role played by the term $S_{B \wedge F}$ in (6.23) in the quantization of electrodynamics. We will do so in two stages. First we will consider the effect of coupling $B$ to the photon in the Coulomb phase of compact electrodynamics, the phase in which the photon is exactly massless. Then we will consider the effect of $B$ in a Higgs phase, and thus arrive at a dual description of quantum hair. 
If we canonically quantize electrodynamics in the temporal $\left(A_{0}=0\right)$ gauge, the coupling to $B$ alters the momentum that is conjugate to the dynamical variable $A_{i}$; the momentum becomes

$$
\Pi^{i}=E^{i}-\frac{e}{2} \epsilon^{i j k} B_{j k}
$$

This modification alters in turn the Gauss's law constraint satisfied by the physical states, and so changes the charge spectrum of the theory. Let us suppose that electrodynamics is compact, meaning that all matter fields have charges that are integer multiples of a fundamental unit, which we will take to be $N e$. Therefore, a global gauge transformation with the gauge function $\lambda=2 \pi / N e$ must act trivially on all states. Now consider a gauge transformation with $\lambda=0$ on the horizon of the black hole, and $\lambda=2 \pi / N e$ at spatial infinity. (The precise form of the transformation between the horizon and infinity is irrelevant, since all gauge transformations of compact support act trivially on physical states.) The action of an infinitesimal gauge transformation on a wave-functional $\Psi[A]$ takes the form

$$
\delta_{\lambda} A_{i}(\vec{x}) \frac{\delta}{\delta A_{i}(\vec{x})}=-\partial_{j} \lambda(\vec{x}) \frac{1}{i \hbar}\left(E^{j}(\vec{x})-\frac{e}{2} \epsilon^{j k l} B_{k l}(\vec{x})\right),
$$

and therefore, the condition for the finite transformation in question to act trivially is

$$
2 \pi i \text { (integer) }=\int d^{3} x\left[-\partial_{j} \lambda(\vec{x}) \frac{1}{i \hbar}\left(E^{j}(\vec{x})-\frac{e}{2} \epsilon^{j k l} B_{k l}(\vec{x})\right)\right] .
$$

Now we may integrate by parts. Since $(7.5)$ must be satisfied by any $\lambda(\vec{x})$ that satisfies the stated boundary conditions, we may discard the resulting volume integral, and retain only the surface term

$$
2 \pi i \text { (integer) }=\frac{2 \pi i}{N \hbar e} \int_{r=\infty} d^{2} S_{j}\left(E^{j}-\frac{e}{2} \epsilon^{j k l} B_{k l}\right) .
$$

We then conclude that

$$
\frac{Q}{N \hbar e}=(\text { integer })+\frac{1}{N \hbar e}\left(e \int_{r=\infty} B\right)
$$


where $Q$ has been defined as the electric flux through the surface at infinity.

Equation (7.7) clarifies the meaning of a $B$ field that is closed but not exact. On a space that contains non-contractible two-spheres (like the exterior region of a black hole) the total charge that determines the behavior of the electric field at infinity is not the same, in general, as the sum of all elementary charges that are contained in the region. There is an additional contribution that is classified by the cohomology of the two form $B$. Clearly, we can and ought to consider the closed part of $B$ as a means of keeping track of the part of the charge that has "fallen in."

In fact, we have also found that the total charge need not be an integer multiple of the elementary charge quantum $N \hbar e$. The charge associated with the $B$ field can have a fractional part that shifts the charge spectrum away from the integers. This phenomenon (and the way that we have derived it) is closely analogous to the corresponding shift in the electric charge spectrum that is induced by a $\theta$-term in the presence of a magnetic monopole. ${ }^{[4]]}$

\subsection{DuAl PiCture of the lasso process}

Now we must understand the effect of the $B$ field in a Higgs phase. The key is provided by (7.7) - the surface integral of $B$ determines the fractional part of the electric charge, the part that cannot be screened by the Higgs condensate. Thus dual variables provide an alternative way to implement a $Z_{N}$ charge projection. Specifying that

$$
\exp \left(\frac{2 \pi i}{N \hbar} \int_{r=\infty} B\right)=e^{i 2 \pi Q / N \hbar e}
$$

is equivalent to weighting the vorticity sectors by appropriate phases, as described earlier. To see this equivalence, it is sufficient to note that the term (6.23) reproduces the desired phase.

It is also enlightening to consider the Lorentzian analog of the vortex - the spacetime process in which a virtual string loop lassoes the black hole and then 
re-annihilates. We wish to see that (6.23) has the effect of weighting this process by an appropriate Aharonov-Bohm phase.

To be definite, suppose that the virtual string sweeps out a sphere that encloses the black hole, and that the $B$ field has the form (7.2). Then we must evaluate

$$
S_{B \wedge F} / \hbar=\frac{Q}{4 \pi \hbar} \int F_{t r} d t d r \sin \theta d \theta d \phi .
$$

Now we recall that, since a static cosmic string has a magnetic field in its core, a string moving at velocity $v$ along the sphere has a radial electric field $F_{t r}$ that is proportional to $v$. Integrating over the cross section of the moving string, we have

$$
\int d y d r F_{t r}=v \Phi
$$

where $\Phi$ is the magnetic flux trapped in the core of the string, and $\hat{y}$ denotes the direction tangent to the sphere and transverse to the string. Since the string moves in the $\hat{y}$ direction at velocity $v$, we may write $d y=v d t$; thus, at each point of the sphere we obtain

$$
\int d t d r F_{t r}=\Phi
$$

if the virtual string sweeps over the sphere exactly once. Doing the $d \theta d \phi$ integral in eq. (7.9), we obtain

$$
S_{B \wedge F} / \hbar=Q \Phi / \hbar
$$

For a string of minimal flux, we have $\Phi=2 \pi / N e$, and so we find

$$
e^{i S_{B \wedge F} / \hbar}=\exp (2 \pi i Q / N \hbar e),
$$

precisely the expected Aharonov-Bohm phase. 
We will briefly describe another way to do the same calculation. First, we note that the electromagnetic field associated with the world sheet of a flux tube of infinitesimal thickness can be written as

$$
F_{\mu \nu}(x)=-\frac{1}{2} \Phi \int \epsilon_{\mu \nu \rho \sigma} \epsilon^{a b} \frac{\partial y^{\rho}}{\partial \xi^{a}} \frac{\partial y^{\sigma}}{\partial \xi^{b}} \delta^{4}(x-y(\xi)) d^{2} \xi
$$

where the coordinates $\left(\xi^{0}, \xi^{1}\right)$ parametrize the world sheet, $y^{\mu}(\xi)$ is the embedding of the world sheet in spacetime, and $\Phi$ is the magnetic flux carried by the string in its rest frame. (Our conventions are such that $\epsilon_{0123}=-1$.) Now, as an example of a world sheet that wraps around the black hole, consider a string that nucleates at the north pole, and annihilates at the south pole, parametrized so that

$$
\begin{aligned}
& \theta=\theta\left(\xi^{0}\right), \\
& \phi=\phi\left(\xi^{1}\right)
\end{aligned}
$$

Then, evaluating eq. (7.9), we have

$$
S_{B \wedge F} / \hbar=\frac{Q \Phi}{4 \pi \hbar} \int \sin \theta \frac{\partial \theta}{\partial \xi_{0}} \frac{\partial \phi}{\partial \xi_{1}} d \xi^{0} d \xi^{1}=Q \Phi / \hbar
$$

We find, again, the desired Aharonov-Bohm phase.

We have shown, then, that the $B \wedge F$ term in the action has the effect of attributing appropriate phases to processes wherein a cosmic string world sheet envelops a charged black hole. We explicitly demonstrated this for a special class of field histories, but the result is general.

\subsection{Global Symmetry AND hair}

In principle one could imagine introducing dual fields for every conserved charge in a theory, whether or not that charge is gauged. Formally then, if the dual field outside a black hole is a closed two-form that is not exact, the black hole "carries" the associated charge. However the question arises, whether the black hole "hair" thus defined is physically observable. 
Indeed the conventional wisdom is quite the contrary, namely that continuous global symmetries are violated (or transcended) by black holes. Indeed, particles that carry globally conserved charges can fall into a black hole. Any information about them that is not associated with a long-range field is completely lost when they impact the singularity. If the black hole has no internal quantum numbers, this process flouts the conservation law, and so the global symmetry loses its power. In particular, if the black hole eventually evaporates completely and disappears, it has no reason to disgorge the same amount of charge as it has swallowed.

Yet it is quite tempting to suggest that our arguments that established the existence of quantum hair in the case where a continuous local symmetry is broken to a nontrivial discrete subgroup can also be applied to the case where a continuous global symmetry symmetry is broken to a nontrivial discrete subgroup. In a sense, the case of a global $U(1)$ symmetry breaking to $Z_{N}$ is just a limiting case of the Higgs mechanism that we have discussed so extensively - namely, the limit in which the gauge coupling $e$ approaches zero. And no $e$ appears in the Aharonov-Bohm phase $\exp (2 \pi i / N)$ that is acquired by a unit charge that circles a minimal string. Indeed, when a global $U(1)$ symmetry is spontaneously broken, global strings arise, and it has recently been shown that (at least in the $Z_{2}$ case) there are infinite range interactions between particles with nontrivial discrete charges and global strings. ${ }^{[48]}$

When a global $U(1)$ symmetry (or any continuous symmetry) is spontaneously broken, the corresponding charge operator $\tilde{Q}$ is ill-defined. But if the objects that condense have charge $N$, then the operator $\exp (2 \pi i \tilde{Q} / \hbar N)$, which acts trivially on the condensate, is well-defined. (Here, $\tilde{Q}$ denotes the classical global charge, so that a "charge-one" particle has $\tilde{Q}=\hbar$.) If the global $Z_{N}$ charge on a black hole can really be measured via an Aharonov-Bohm interaction with a global string, then the same arguments that we used in Section 2 would seem to imply that black holes can carry global $Z_{N}$ hair. It would be most peculiar if this were so, for it would mean that the breaking of the continuous symmetry has rendered black holes, which previously transcended global charge conservation, suddenly more fastidious. Perhaps fortunately, there are good reasons, both formal and physical, 
to believe that quantum hair associated with global symmetries does not exist.

1. The partition function of a black hole with specified global $Z_{N}$ charge is given by a formula like eq. (4.2), but with $Q / e$ replaced by the global charge $\tilde{Q}$ of the black hole, and where $Z_{k}$ is the path integral in the sector with global vorticity $k$; i.e. such that the phase of the condensate rotates by $-2 \pi k$ as $\tau$ varies from 0 to $\beta$. Now, however, on a fixed asymptotically flat background geometry there are no vortex configurations of finite action in the infinite volume limit; in fact, the action of a vortex diverges like $R^{3}$ with the radius $R$. Things are even worse for a vortex coupled to gravity - there are no asymptotically flat solutions at all. If we assume that such a solution exists, then we can compute that $T_{\mu}^{\mu}=T_{\tau \tau}$ is a non-zero constant at infinity, contradicting the Einstein equations. We conclude, then, that the $k \neq 0$ contribution to the partition function is snuffed out, and with it the charge dependence. (It may seem odd that global vortices on the Euclidean black hole background do not exist, since there appears to be no reason why a virtual global string cannot wind around the horizon of a hole. But in fact, this winding would introduce a physical twist in the Goldstone boson field that would asymptotically approach the horizon at large Schwarzschild time. The string must wind back the other way to return the black hole to its initial state.)

2. Alternatively, we can perform the projection onto a sector of specified $Z_{N}$ charge by fixing the surface integral of the dual two-form $B$, according to

$$
\exp \left(\frac{i}{N \hbar} \int_{r=\infty} B\right)=e^{2 \pi i \tilde{Q} / \hbar N}
$$

Now, when the $Z_{N}$ symmetry is gauged, we have seen that the cohomology of $B$ can have a non-trivial effect that arises from the $B \wedge F$ term in the action. But if the $Z_{N}$ symmetry is not gauged, then $B$ enters the action only through the field strength $H=d B$ - the closed part of $B$ is completely 
decoupled. Thus, in a different way, we see that the partition function is independent of the charge.

3. The thought-experimental method of measuring the charge by scattering global strings off a hole, and looking for an Aharonov-Bohm type contribution to the cross section, is less than convincing. First of all, since there is no mass gap in the theory, the strings are not well localized, and even distant strings (i.e. with distant cores) have local interactions with the hole. Since the low-energy, forward contribution to the cross-section we seek involves slow motion of the hole past the string, there is every opportunity for the black hole to disrupt or even swallow the string. Secondly, the AharonovBohm behavior of the scattering of a string by a $Z_{2}$ charge arises only if the passage of the string by the charge can be regarded as adiabatic, as described in Ref. 48. But if we consider a $Z_{2}$ charge in the vicinity of a black hole, this adiabatic condition becomes more and more stringent as the charge gets closer and closer to the horizon, because of the gravitational time dilation. Thus, the long-range interaction between charge and string is destroyed as the charge falls into the black hole.

4. A revealing contrast between gauged and global discrete symmetries is found when one attempts to construct an operator that measures discrete global charge. In the case of a local $Z_{N}$ symmetry, it is possible to construct an operator that has support on a closed surface $\Sigma$, such that the phase of the expectation value of this operator is sensitive to the $Z_{N}$ charge enclosed by $\Sigma^{[22,23]}$ The existence of this operator shows that the $Z_{N}$ charge cannot disappear, and so black holes must be capable of carrying such charges. But the attempt to construct the corresponding operator for global $Z_{N}$ charge meets an obstacle. In the dual language, the candidate charge operator can be expressed as

$$
\frac{\exp \left(\frac{2 \pi i}{N \hbar} \int_{\Sigma} B\right)}{\left\langle\exp \left(\frac{2 \pi i}{N \hbar} \int_{\Sigma} B\right)\right\rangle_{0}} .
$$

Physically, we may envision this operator as an insertion of a classical global 
string on the world sheet $\Sigma$, and its expectation value may be interpreted as the Aharonov-Bohm phase acquired by this string as it winds around the region bounded by $\Sigma{ }^{[23]}$ Because the string is poorly localized, however, the action of the string increases with the linear size $r$ of $\Sigma$ like $r^{2} \log (r)$. Hence, it is favorable for a dynamical global string to nucleate in order to shield the long-range effect of the classical string; then the action is of order $r^{2}$ (without the logarithm). This means that the charge deep inside a large region is not detected. In contrast to the situation when the symmetry is gauged, this construction does not force us to conclude that the charge must be conserved when black holes are present.

Finally let us add a few words concerning axions. The first remark to be made is that the valid form of B-hair, as we have discussed it above, has nothing to do with axions. The coupling of axions to the gauge field is quite different from the $B \wedge F$ term. That term, from which we derived our hair, is inextricably linked with discrete gauge symmetries. Whether the gauge symmetries are introduced in the familiar form of $J \cdot A$ coupling, or in the less familiar form of the $B \wedge F$ term makes no fundamental difference, since these are mathematically equivalent.

Even if the $B \wedge F$ interaction is postulated as a fundamental property of strings, which is the minimum necessary to reproduce the result of the previous section, the interpretation of B-hair as discrete gauge hair seems to follow ineluctably. For example, in previous chapters we have seen in detail how effective electric fields around black holes are built up from the virtual string looping process. This provides a strong hint that one may work backwards, and construct an effective gauge theory as a consequence of the existence of the postulated strings and couplings. 


\section{Primary and Secondary Hair}

The classic no-hair theorems can be interpreted in two senses. A strong interpretation of these theorems would say that they require that black holes settle down precisely to the classic known forms (Kerr-Newman) characterized completely by mass, angular momentum, and charge. According to this strong interpretation there can be no non-trivial fields outside the black hole aside from the gravitational and electromagnetic (or more general continuous gauge) fields.

This strong interpretation of the no-hair theorem, however, is readily seen to be violated in a number of circumstances. An example that has been much discussed recently is the dilaton black hole..$^{[9,10,49-51]}$ Motivated by considerations in string theory, one considers adding to the Einstein Lagrangian additional terms of the form

$$
\mathcal{L}_{\text {dil. }}=\sqrt{g}\left(\frac{1}{2} g^{\mu \nu} \partial_{\mu} \phi \partial_{\nu} \phi-\frac{1}{4} e^{-2 a \phi} g^{\mu \rho} g^{\nu \sigma} F_{\mu \nu} F_{\rho \sigma}\right)
$$

This reduces to the standard Einstein-Maxwell form if $\phi=$ constant . However if $g^{\mu \rho} g^{\nu \sigma} F_{\mu \nu} F_{\rho \sigma} \neq 0$, such as occurs outside a charged black hole, then the field equation for $\phi$ will not be satisfied by a constant - the non-vanishing electromagnetic field acts a source for the dilaton field $\phi$. Similarly an axion field, which couples linearly to $\epsilon^{\mu \nu \rho \sigma} F_{\mu \nu} F_{\rho \sigma}$, will necessarily vary, and be non-trivial, outside a dyonic hole which carries both electric and magnetic charge. ${ }^{[50,52]}$ Yet another example is the recently discovered black hole embedded in a magnetic monopole, ${ }^{[36]}$ which has a Higgs field core outside the horizon.

We would like to call this sort of hair, which is generated because the basic fields (associated with mass, angular momentum, and continuous gauge charges) act as sources for other fields, secondary hair. ${ }^{[26]}$

Actually, we need not invoke exotic particles to construct examples of secondary hair. It occurs in the standard model, when perturbative corrections to the matter Lagrangian are taken into account. For example, perturbative corrections to the 
effective Lagrangian for the standard model can hardly fail to generate terms of the form

$$
\Delta \mathcal{L} \propto \sqrt{g} F_{\alpha \beta} R_{\rho \sigma \tau}^{\alpha} R_{\lambda \eta}^{\beta \rho} \epsilon^{\sigma \tau \lambda \eta}
$$

Such a term will act as a source term for the electromagnetic field in the presence of suitable curvature. It will, for example, generate a non-vanishing electric field outside a rotating electrically neutral black hole: essentially, an electric dipole moment. (Note that for a non-rotating neutral black hole with no other additive quantum numbers, the existence of an electric field outside is forbidden by CPT. The correlation of an electric dipole moment with spin direction is $\mathrm{P}$ and $\mathrm{T}$ violating, as of course is the interaction (8.2) responsible for it.) Also, the magnitude of the Higgs field will not be quite constant outside the hole, for similar reasons. Let $\eta$ denote the Higgs field and $v$ its expectation value, with $\eta \equiv v+\zeta$. Terms in the effective Lagrangian of the form

$$
\Delta \mathcal{L} \propto \sqrt{g}|\eta|^{2} R_{\mu \nu \rho \sigma} R^{\mu \nu \rho \sigma} \rightarrow 2 \sqrt{g} v \zeta R_{\mu \nu \rho \sigma} R^{\mu \nu \rho \sigma}+\ldots
$$

then act as a linear source for $\zeta$ in the presence of curvature, so $\zeta$ will certainly vary in a non-trivial way outside the hole.

Another, weaker but more profound way of interpreting the no-hair theorems is as statements about the classification of stationary black holes. According to this weaker interpretation, the properties of a black hole are completely determined, within any given theory, by the value of its mass, angular momentum, and continuous gauge charges. As we have seen, this weaker interpretation is violated non-perturbatively in $\hbar$, by discrete gauge hair. This form of hair expands the space of states of black holes. It is therefore appropriately called primary hair.

In the classical analysis of the field equations, which inspires the no-hair theorems, linear perturbation theory suggests that a massless (integer) spin $s$ field can support hair in partial waves $l \leq(s-1)$. Associated with the spin-2 graviton, then, we have hair in partial waves $l=0$ (namely $M$ ) and $l=1(J)$; associated with 
the spin-1 photon, we have hair in $l=0(Q)$. We have shown that the restriction to massless fields can be removed in the case $s=1$. It will be interesting to see whether this result can be extended to symmetries associated with higher spins, such as are suggested by superstring theory. If so, then there are real prospects that the complete internal state of black holes (including the wealth of states indicated by the area law for large black holes) might be accounted for.

After this work was completed, we learned that Dowker, Gregory, and Traschen ${ }^{[53]}$ have also investigated the properties of the Euclidean vortex solutions that we discussed in Section 4.

Acknowledgements: We gratefully acknowledge helpful discussions with Mark Alford, Martin Bucher, Gary Gibbons, Stephen Hawking, Lawrence Krauss, John March-Russell, Alex Ridgway, Andy Strominger, Kip Thorne, Alex Vilenkin, and Edward Witten.

\section{REFERENCES}

1. S. W. Hawking, Phys. Rev. D14 (1976) 2460.

2. S. W. Hawking, Comm. Math. Phys. 43 (1975) 199.

3. J. D. Bekenstein, Phys. Rev. D7 (1973) 2333.

4. See, for example, R. Wald, General Relativity (University of Chicago Press, Chicago, 1984).

5. J. D. Bekenstein, Phys. Rev. D5 (1972) 1239, 2403

6. C. Teitelboim, Phys. Rev. D5 (1972) 2941.

7. S. W. Hawking, Phys. Rev. D13 (1976) 191.

8. G. 't Hooft, Nucl. Phys. B335 (1990) 138.

9. G. W. Gibbons, and K. Maeda, Nucl. Phys. B298 (1988) 741. 
10. D. Garfinkle, G. T. Horowitz, and A. Strominger, Phys. Rev. D43 (1991) 3140.

11. V. Rubakov, JETP Lett. 33 (1981) 644; C. Callan, Phys. Rev. D25 (1982) 2141.

12. S. W. Hawking, Phys. Rev. D37 (1988) 904; S. W. Hawking and R. Laflamme, Phys. Lett. 209B (1988) 39.

13. S. Coleman, Nucl. Phys. B307 (1988) 867; B310 (1988) 643.

14. S. Giddings and A. Strominger, Nucl. Phys. B307 (1988) 854.

15. S. W. Hawking, Mod. Phys. Lett A5 (1990) 145, 453.

16. R. H. Price, Phys. Rev. D5 (1972) 2419, 2439.

17. E. Newman, R. Penrose, J. Math. Phys. 3 (1962) 566.

18. L. M. Krauss and F. Wilczek, Phys. Rev. Lett. 62 (1989) 1221.

19. R. Rohm, Princeton University Ph.D. thesis (1985) unpublished; M. G. Alford and F. Wilczek, Phys. Rev. Lett. 62 (1989) 1071.

20. Y. Aharonov and D. Bohm, Phys. Rev. 119 (1959) 485.

21. M. J. Bowick, S. B. Giddings, J. A. Harvey, G. T. Horowitz, and A. Strominger, Phys. Rev. Lett. 61 (1988) 2823.

22. M. G. Alford, J. March-Russell, and F. Wilczek, Nucl. Phys. B337 (1990) 695.

23. J. Preskill and L. M. Krauss, Nucl. Phys. B341 (1990) 50.

24. J. Preskill, Phys. Scrip. T36 (1991) 258.

25. S. Coleman, J. Preskill, and F. Wilczek, Mod. Phys. Lett. A6 (1991) 1631.

26. S. Coleman, J. Preskill, and F. Wilczek, Phys. Rev. Lett. 67 (1991) 1975.

27. E. J. Weinberg, D. London, and J. Rosner, Nucl. Phys. B236 (1984) 90. 
28. A. M. Polyakov, JETP Lett. 20 (1974) 194; G. 't Hooft, Nucl. Phys. B79 (1974) 276.

29. S. Coleman, in The Unity of the Fundamental Interactions, ed. A. Zichichi (Plenum, New York, 1983).

30. M. Srednicki and L. Susskind, Nucl. Phys. B179 (1981) 239.

31. D. J. Gross, R. D. Pisarski, and L. G. Yaffe, Rev. Mod. Phys. 53 (1981) 43.

32. G. W. Gibbons and S. W. Hawking, Phys. Rev. D15 (1977) 2752.

33. G. W. Gibbons, Comm. Math. Phys. 44 (1975) 245.

34. G. W. Gibbons and C. M. Hull, Phys. Lett. 109B (1982) 190.

35. F. A. Bais and R. J. Russell, Phys. Rev. D11 (1975) 2692; Y. M. Cho and P. G. O. Freund, Phys. Rev. D12 (1975) 1588.

36. K. Lee, V. P. Nair, and E. J. Weinberg, "Black Holes in Magnetic Monopoles," Columbia preprint CU-TP-539 (1991); "A Classical Instability of Reissner-Nordström Solutions and the Fate of Magnetically Charged Black Holes," Columbia preprint CU-TP-540 (1991).

37. R. Brandt and F. Neri, Nucl. Phys. B161 (1979) 253.

38. A. K. Gupta, J. Hughes, J. Preskill, and M. B. Wise, Nucl. Phys. B333 (1990) 195.

39. M. Bucher, K.-M. Lee, and J. Preskill, "On Detecting Discrete Cheshire Charge," Caltech preprint CALT-68-1753 (1991).

40. E. B. Bogomol'nyi, Sov. J. Nucl. Phys. 24 (1976) 449.

41. A. Vilenkin, Phys. Rev. D23 (1981) 852.

42. S. L. Adler and R. B. Pearson, Phys. Rev. D18 (1978) 2798.

43. G. W. Gibbons, "Self-Gravitating Magnetic Monopoles, Global Monopoles and Black Holes," Lectures at the 1990 Lisbon Autumn School, DAMTP R90/31 (1990). 
44. G. 't Hooft, Nucl. Phys. B153 (1979) 141.

45. M. B. Green and J. H. Schwarz, Phys. Lett. 149B (1984) 117.

46. T. J. Allen, M. J. Bowick, and A. Lahiri, Phys. Lett. B237 (1990) 47.

47. E. Witten, Phys. Lett. 86B (1979) 283.

48. J. March-Russell, J. Preskill, and F. Wilczek, "Internal Frame Dragging and a Global Analogue of the Aharonov-Bohm Effect," Princeton preprint PUPT-91-1297 (1991).

49. J. Preskill, P. Schwarz, A. Shapere, S. Trivedi, and F. Wilczek, Mod. Phys. Lett. A6 (1991) 2353.

50. A. Shapere, S. Trivedi, and A. Shapere, Mod. Phys. Lett. A6 (1991) 2677.

51. C. F. E. Holzhey and F. Wilczek, "Black Holes as Elementary Particles," IASSNS-HEP-91/71 (1991).

52. B. A. Campbell, N. Kaloper, and K. A. Olive, Phys. Lett. B263 (1991) 364; B. A. Campbell, M. J. Duncan, N. Kaloper, and K. A. Olive, Phys. Lett. B251 (1990) 34; K. Lee and E. J. Weinberg, "Charged Black Holes with Scalar Hair," Columbia preprint CU-TP-515 (1991).

53. F. Dowker, R. Gregory, and J. Traschen, "Euclidean Black Hole Vortices," FERMILAB-Pub-91/332-A (1991). 


\section{FIGURE CAPTIONS}

1) The causal structure of a classical Schwarzschild black hole formed by collapse is clearly displayed in its Penrose diagram, (a). The distant future outside the black hole, represented by the solid boundary, is not complete, in the sense that knowledge of the wave function on this surface does not allow one to construct it throughout space-time. It could be completed by including the horizon, or the singularity (dotted lines). Thus one could have a unique, unitary connection between the wave function given in the distant past before the collapse and the wave function on either completion. However if we lose contact with our friendly observers on the horizon, or at the singularity, information is lost and the evolution appears not to be unitary. The problem is especially acute if we imagine that the black hole, after formation, eventually evaporates completely - for then these observers must eventually cease to exist, as shown in (b). 